\title{
SMALL BIALGEBRAS WITH A PROJECTION: APPLICATIONS
}

\author{
A. ARDIZZONI AND C. MENINI
}

\begin{abstract}
In this paper we continue the investigation started in AMStu, dealing with bialgebras $A$ with an $H$-bilinear coalgebra projection over an arbitrary subbialgebra $H$ with antipode. These bialgebras can be described as deformed bosonizations $R \# \xi H$ of a pre-bialgebra $R$ by $H$ with a cocycle $\xi$. Here we describe the behavior of $\xi$ in the case when $R$ is f.d. and thin i.e. it is connected with one dimensional space of primitive elements. This is used to analyze the arithmetic properties of $A$. Meaningful results are obtained when $H$ is cosemisimple. By means of Ore extension construction, we provide some examples of atypical situations (e.g. the multiplication of $R$ is not $H$-colinear or $\xi$ is non-trivial).
\end{abstract}

\section{CONTEnTS}

Introduction

1. Preliminaries

2. The cocycle

3. Ad-invariant integrals

4. Ore Extensions

5. Compatible Data

6. Examples

References

\section{INTRODUCTION}

Let $A$ be a bialgebra and assume that the coradical $H$ of $A$ is a subbialgebra of $A$ with antipode i.e. that $A$ has the so-called dual Chevalley property.

By using the Hochschild cohomology in monoidal categories, it was proved in AMSte, Theorem 2.35] that the canonical injection of $H$ in $A$ has a retraction $\pi: A \rightarrow H$ which is an $H$-bilinear coalgebra map. This led to the investigation of the structures of bialgebras $A$ with an $H$-bilinear coalgebra projection onto an arbitrary subbialgebra $H$ with antipode. There is a full description of these structures in terms of pre-bialgebras in ${ }_{H}^{H} \mathcal{Y D}$ with a cocycle (called dual Yetter-Drinfeld quadruples in AMSt, Definition 3.59]) and a bosonization type procedure. Namely (see AMSte, Theorem 3.64]) to such an $A$ one associates a 5-tuple $(R, m, u, \delta, \varepsilon)$ (called pre-bialgebra), where $\left(R=A^{C o(H)}, \delta, \varepsilon\right)$ is a coalgebra in the category $\left({ }_{H}^{H} \mathcal{Y D}, \otimes, K\right), u: K \rightarrow R, m: R \otimes R \rightarrow R$ are $K$-linear maps satisfying five equalities (see Definition 1.3) which make $R$ a sort of unital bialgebra in ${ }_{H}^{H} \mathcal{Y D}$ with the following differences: the multiplication is not necessarily associative neither a morphism of $H$-comodules. This particular pre-bialgebra is also endowed with a $K$-linear map $\xi: R \otimes R \rightarrow H$ (called corresponding cocycle) which fulfills six equalities (see Definition 1.10). Then $A$ can be reconstructed by these data. In fact the bialgebra $A$ is isomorphic to $R \#_{\xi} H$ which is $R \otimes H$ endowed with a suitable bialgebra structure that depends on the pre-bialgebra and the corresponding cocycle: this structure on $R \otimes H$ can be somehow regarded as a deformation of the usual bosonization structure (see [Rad and Ma]) via $\xi$. In [AMStu], we proposed to describe

1991 Mathematics Subject Classification. Primary 16W30; Secondary 16S40.

Key words and phrases. Hopf algebras, bialgebras, bosonizations.

This paper was written while both the authors were members of G.N.S.A.G.A. with partial financial support from M.I.U.R.. 
the bialgebra structure of $R \#_{\xi} H$. We did a first step by considering the case when the coalgebra $R$ is $N$-dimensional and thin i.e. it is connected and the space of its primitive elements is one dimensional generated by $y \in R$. It turned out that $R$, which usually carries a non-associative multiplication, is in fact an associative $K$-algebra but not a braided bialgebra in ${ }_{H}^{H} \mathcal{Y} \mathcal{D}$. By means of this achievement, we proved the main results of AMStu. Explicitly we completely described the bialgebra structure of $A$ whenever $H$ is either f.d. or cosemisimple. This new description allowed us to construct another projection of $A$ onto $H$ which is normalized in the sense that it gives rise to a new pre-bialgebra $(R, m, u, \delta, \varepsilon)$ which is now a braided bialgebra in the category $\left({ }_{H}^{H} \mathcal{Y} \mathcal{D}, \otimes, K\right)$ and in fact a quantum line (but still with a not necessarily trivial corresponding cocycle).

In this paper (see Section 2) we investigate the properties of $\xi$ for a generic projection. Let $0 \leq a, b \leq N-1$. We know that

$$
\xi\left(y^{a} \otimes y^{b}\right)=0 \text { unless } a+b=0, \frac{N}{2}, N, \frac{3 N}{2}
$$

whenever this makes sense. In Proposition 2.8, we prove that $\xi\left(y^{a} \otimes y^{b}\right)$ is always constant on the line $a+b=N / 2$. It is also constant on the line $a+b=N$ (respectively $a+b=3 N / 2$ ) whenever $\left[\xi\left(y \otimes y^{N / 2-1}\right)\right]^{2}=0$ (respectively $\xi\left(y \otimes y^{N / 2-1}\right)=0$ ). When $H$ is finitely dimensional or cosemisimple there is a primitive $\theta$-th root of unity $q \in K$, where $2 \leq \theta \leq N$, and $g \in H, \chi \in H^{*}$ and $\lambda(N) \in K$ such that $\xi\left(y \otimes y^{N-1}\right)=\xi\left(y^{2} \otimes y^{N-2}\right)=\cdots=\xi\left(y^{N-1} \otimes y\right)=\lambda(N)\left(1_{H}-g^{N}\right)$. Moreover $(H, g, \chi, \lambda(N))$ is a compatible datum for $q$ (see Proposition 2.11) and the cocycle is completely described whenever $\xi\left(y \otimes y^{N / 2-1}\right)=0$. In Theorem 2.14, we prove that the following assertions are equivalent:

(a) $m$ is left $H$-colinear;

(b) $N$ is odd or $\xi\left(y \otimes y^{N / 2-1}\right)=0$;

(c) The $n$-th iterated power $y^{\cdot{ }^{n} n}$ of $y$ in $R$ and the $n$-th iterated power $y^{\cdot A n}$ of $y$ in $A$ coincides for every $0 \leq n \leq N-1$;

(d) $R=R_{q}(H, g, \chi)$ is a quantum line.

Furthermore we characterize when the bosonization $R \#_{\xi} H$ is a Radford-Majid bosonization.

In Section 3 , we apply the foregoing results in the case when $H$ is a Hopf algebra endowed with a so-called $a d$-invariant integral. In particular, in Proposition 3.7, we prove that if $\xi \neq \varepsilon \otimes \varepsilon$, then we have $\chi^{N}=\varepsilon_{H}$ and $g^{N} \in Z(H) \backslash\left\{1_{H}\right\}$.

When $H$ is cosemisimple (i.e. $H$ is the coradical of $A$ ), we show (see Corollary 4.11) that there exists a unique $H$-bilinear projection onto $H$. By the foregoing, this projection must be normalized so that the associated pre-bialgebra is always a quantum line.

Let $A$ be a finite dimensional bialgebra over a field $K$. Suppose that the coradical $H$ of $A$ is a subbialgebra of $A$ with antipode. Then $A$ is a Hopf algebra and, as recalled above, there is a retraction $\pi: A \rightarrow H$ (i.e. $\pi \sigma=H$ where $\sigma: H \rightarrow A$ denotes the canonical injection) that is an $H$-bilinear coalgebra map. Let $(R, m, u, \delta, \varepsilon)$ be the pre-bialgebra in ${ }_{H}^{H} \mathcal{Y D}$ associated to $(A, \pi, \sigma)$ with corresponding cocycle $\xi$. Then in Theorem 4.5, we proved that $R$ is thin if and only if $\operatorname{dim} A_{1}=2 \operatorname{dim} H$. Part of this result is contained in [AS, Corollary, page 673] where it is proved that if $\operatorname{dim} A_{1}=2 \operatorname{dim} H$, then $A$ is generated as an algebra by $A_{1}$.

In order to completely describe a bialgebra $A$ as above, for an arbitrary compatible datum $(H, g, \chi, \lambda(N))$ (see Definition 1.6) we construct a Hopf algebra $\mathcal{O}(H, g, \chi, \lambda(N)$ ) (see Theorem 4.2 which is endowed with a normalized projection onto $H$. Furthermore, we prove the following result (see Theorem 4.4). Assume that $H$ is either a f.d. or cosemisimple Hopf algebra over a field $K$ and $A$ is a bialgebra endowed with an injective morphism of bialgebras $\sigma: H \rightarrow A$ having a retraction $\pi: A \rightarrow H$ (i.e. $\pi \sigma=H$ ) that is an $H$-bilinear coalgebra map. If the underlying coalgebra in the pre-bialgebra in ${ }_{H}^{H} \mathcal{Y D}$ associated to $(A, \pi, \sigma)$ is $N$-dimensional and thin, then $A \simeq \mathcal{O}(H, g, \chi, \lambda(N))$ as a bialgebra for a suitable a compatible datum $(H, g, \chi, \lambda(N))$. Note that this holds in the particular case when $A$ is a finite dimensional bialgebra with the dual Chevalley property such that $\operatorname{dim} A_{1}=2 \operatorname{dim} A_{0}$. 
These results will enable us to construct some interesting examples. In particular an example of a Hopf algebra of dimension 72 with a non normalized projection will be given in Example 6.4. This can be proved to be of minimal dimension with respect to this property (see Remark 6.3). We also provide a minimal example where the pre-bialgebra is a quantum line but the bosonization is not a Radford-Majid bosonization (see Remark Theorem 6.5). An example of this situation where $H$ is infinite dimensional is given in Example 6.1. In these two last examples $H$ comes out to be cosemisimple. Thus, by Corollary 1.10, they can not be regarded as Radford-Majid bosonizations of the Hopf subalgebra $H$ for some other set of compatible data.

We assume for simplicity of the exposition that our ground field $K$ has characteristic 0 . Anyway we point out that many results below are valid under weaker hypotheses.

\section{Preliminaries}

Let $H$ be a Hopf algebra over the field $K$. Recall that an object $V$ in ${ }_{H}^{H} \mathcal{Y D}$ is a left $H$-module and a left $H$-comodule satisfying, for any $h \in H, v \in V$, one of the following equivalent compatibility conditions:

$$
\begin{gathered}
\sum\left(h_{(1)} v\right)_{<-1>} h_{(2)} \otimes\left(h_{(1)} v\right)_{<0>}=\sum h_{(1)} v_{<-1>} \otimes h_{(2)} v_{<0>}, \\
\rho(h v)=\sum h_{(1)} v_{<-1>} S\left(h_{(3)}\right) \otimes h_{(2)} v_{<0>},
\end{gathered}
$$

where $\rho: V \rightarrow H \otimes V$ is the coaction of $H$ on $V$ and for the action of $H$ on $V$ we used the notation $h v$, for every $h \in H, v \in V$. If there is danger of confusion we write ${ }^{h} v$ instead of $h v$.

The tensor product $V \otimes W$ of two Yetter-Drinfeld modules is an object in ${ }_{H}^{H} \mathcal{Y D}$ via the diagonal action and the codiagonal coaction; the unit in ${ }_{H}^{H} \mathcal{Y D}$ is $K$ regarded as a left $H$-comodule via the map $x \mapsto 1_{H} \otimes x$ and as a left $H$-module via $\varepsilon_{H}$. Recall that, for every $V, W \in{ }_{H}^{H} \mathcal{Y D}$ the braiding is given by:

$$
c_{V, W}: V \otimes W \rightarrow W \otimes V, \quad c_{V, W}(v \otimes w)=\sum v_{\langle-1\rangle} w \otimes v_{\langle 0\rangle} .
$$

If $H$ has bijective antipode, then $\left({ }_{H}^{H} \mathcal{Y D}, c\right)$ is a braided category.

1.1. Let $R$ and $S$ be two algebras in the braided category ${ }_{H}^{H} \mathcal{Y D}$. We can define a new algebra structure on $R \otimes S$, by using the braiding (11), and not the usual flip morphism. The multiplication in this case is defined by the formula:

$$
(r \otimes s)(t \otimes v)=\sum r\left(s_{\langle-1\rangle} t\right) \otimes s_{\langle 0\rangle} v .
$$

Let us remark that, for any algebra $R \operatorname{in}_{H}^{H} \mathcal{Y D}$, the smash product $R \# H$ is a particular case of this construction. Just take $S=H$ endowed with the left adjoint action (i.e. ${ }^{h} x=\sum h_{(1)} x S\left(h_{(2)}\right)$, for every $h, x \in H$ ) and the usual left $H$-comodule structure.

1.2. Let $R$ and $S$ be two coalgebras in the braided category ${ }_{H}^{H} \mathcal{Y D}$. We can define a new coalgebra structure on $R \otimes S$, by using the braiding (11), and not the usual flip morphism. The comultiplication in this case is defined by the formula:

$$
\delta_{R \otimes S}(r \otimes s)=\sum r^{(1)} \otimes r_{\langle-1\rangle}^{(2)} s^{(1)} \otimes r_{\langle 0\rangle}^{(2)} \otimes s^{(2)} .
$$

Let us remark that, for any coalgebra $R$ in ${ }_{H}^{H} \mathcal{Y D}$, the smash coproduct $R \# H$ is a particular case of this construction. Just take $S=H$ endowed with the left adjoint coaction (i.e. $\rho(h)=$ $\sum h_{(1)} S\left(h_{(3)}\right) \otimes h_{(2)}$, for every $\left.h \in H\right)$ and the usual left $H$-module structure.

Definition 1.3. AMStu, Definition 2.3] Let $H$ be a Hopf algebra. A pre-bialgebra $(R, m, u, \delta, \varepsilon)$ in ${ }_{H}^{H} \mathcal{Y D}$ consists of

- a coalgebra $(R, \delta, \varepsilon)$ in the category $\left({ }_{H}^{H} \mathcal{Y D}, \otimes, K\right)$. 
- two $K$-linear maps

$$
m: R \otimes R \rightarrow R \quad \text { and } \quad u: K \rightarrow R
$$

such that, for all $r, s \in R$ and $h \in H$, the following relations are satisfied:

$$
\begin{aligned}
& h \cdot u(1)=\varepsilon_{H}(h) u(1) \quad \text { and } \quad \rho_{R} u(1)=1_{H} \otimes u(1) \\
& \delta u(1)=u(1) \otimes u(1) \quad \text { and } \quad \varepsilon u(1)=1_{K} ; \\
& h m_{R}(r \otimes s)=\sum m_{R}\left(h_{(1)} r \otimes h_{(2)} s\right) ; \\
& \delta m_{R}=\left(m_{R} \otimes m_{R}\right) \delta_{R \otimes R} \quad \text { and } \quad \varepsilon m_{R}=m_{K}(\varepsilon \otimes \varepsilon) ; \\
& m_{R}(R \otimes u)=R=m_{R}(u \otimes R) ;
\end{aligned}
$$

Note that (3) and (4) mean that $u$ is a coalgebra homomorphism in ${ }_{H}^{H} \mathcal{Y D}$, (5) and (6) mean that $m_{R}$ is left $H$-linear coalgebra homomorphism while (7) means that $u$ is a unit for $m_{R}$. We fix the following notation

$$
\delta(r)=\sum r^{(1)} \otimes r^{(2)}, \text { for every } r \in R .
$$

A morphism $f:(R, m, u, \delta, \varepsilon) \rightarrow\left(R^{\prime}, m^{\prime}, u^{\prime}, \delta^{\prime}, \varepsilon^{\prime}\right)$ of pre-bialgebras in ${ }_{H}^{H} \mathcal{Y D}$ is a coalgebra homomorphism $f:(R, \delta, \varepsilon) \rightarrow\left(R^{\prime}, \delta^{\prime}, \varepsilon^{\prime}\right)$ in the category $\left.{ }_{H}^{H} \mathcal{Y D}, \otimes, K\right)$ such that

$$
f \circ m=m^{\prime} \circ(f \otimes f) \quad \text { and } \quad f \circ u=u^{\prime}
$$

i.e. $f$ is also a homomorphism of non-associative algebras.

REMARK 1.4. To explain the meaning of the concept of pre-bialgebra in ${ }_{H}^{H} \mathcal{Y D}$, it is useful to compare it with the concept of a bialgebra in ${ }_{H}^{H} \mathcal{Y} \mathcal{D}$. A pre-bialgebra is just a unital bialgebra in ${ }_{H}^{H} \mathcal{Y D}$ with the following differences:

a) the multiplication is not necessarily associative;

b) the multiplication is not necessarily a morphism of $H$-comodules.

Definitions 1.5. Let $q$ be a primitive $N$-th root of unity. Let $H$ be a Hopf algebra, $g \in H$ and $\chi \in H^{*}$.

Following CDMM, Definition 2.1], we say that $(H, g, \chi)$ is a Yetter-Drinfeld datum for $q$ whenever

- $g \in G(H)$,

- $\chi \in H^{*}$ is a character of $H$,

- $\chi(g)=q$,

- the following relation holds true

$$
g \sum \chi\left(h_{(1)}\right) h_{(2)}=\sum h_{(1)} \chi\left(h_{(2)}\right) g .
$$

If $(H, g, \chi)$ is a Yetter-Drinfeld datum for $q$, we denote by $R_{q}$ the graded algebra $K[X] /\left(X^{N}\right)$. Let $y=X+\left(X^{N}\right)$. Then $R_{q}$ can be endowed with a unique braided bialgebra structure in $\left({ }_{H}^{H} \mathcal{Y} \mathcal{D}, \otimes, K\right)$, where the Yetter-Drinfeld module structure is given by

$$
h y=\chi(h) y \quad \text { and } \quad \rho(y)=g \otimes y
$$

and the coalgebra structure is defined by setting

$$
\delta(y)=y \otimes 1+1 \otimes y .
$$

In this way $R_{q}$ becomes a braided Hopf algebra that will be denoted by $R_{q}(H, g, \chi)$ and called a quantum line (see $\mathrm{AS}$ ).

Definition 1.6. Let $q$ be a primitive $N$-th root of unity. A compatible datum for $q$ is a quadruple $(H, g, \chi, \lambda(N))$, where

- $(H, g, \chi)$ is a Yetter-Drinfeld datum for $q$, 
- $\lambda(N) \in K$ and $\lambda(N)=0$ if

$g^{N}=1_{H}, \quad$ or $\quad \chi^{N}(h)\left(1_{H}-g^{N}\right) \neq \sum h_{(1)}\left(1_{H}-g^{N}\right) S\left(h_{(2)}\right)$, for some $h \in H$,

while $\lambda(N)$ is arbitrary otherwise.

A compatible datum is called trivial whenever $\lambda(N)=0$ and it is called non-trivial otherwise.

Definition 1.7. We will say that a $K$-coalgebra $C$ is a thin coalgebra whenever

$$
\operatorname{dim}_{K} C_{0}=1 \quad \text { and } \quad \operatorname{dim}_{K} P(C)=1,
$$

where $C_{0}$ denotes the coradical of $C$ and $P(C)$ is the space of primitive elements of $C$.

Lemma 1.8. AMSty, Lemma 2.7] Let $H$ be a Hopf algebra and let $(R, m, u, \delta, \varepsilon)$ be a finite dimensional pre-bialgebra in ${ }_{H}^{H} \mathcal{Y D}$. Assume that $R$ is a thin coalgebra where $P(R)=K y$. Then there is a primitive $\theta$-th root of unity $q \in K$, where $2 \leq \theta \leq \operatorname{dim}_{K}(R)$, and $g \in H, \chi \in H^{*}$ such that

1) $(H, g, \chi)$ is a Yetter-Drinfeld datum for $q$,

2) ${ }^{H} \rho_{R}(y)=g \otimes y$,

3) $h y=\chi(h) y$ for every $h \in H$.

Definition 1.9. Let $H$ be a Hopf algebra and let $(R, m, u, \delta, \varepsilon)$ be a finite dimensional prebialgebra in ${ }_{H}^{H} \mathcal{Y} \mathcal{D}$. Assume that $R$ is a thin coalgebra and let $P(R)=K y$. Consider $q$ and the Yetter-Drinfeld datum $(H, g, \chi)$ for $q$ as in Lemma 1.8. Then $(H, g, \chi)$ will be called the YetterDrinfeld datum associated to the pre-bialgebra $(R, m, u, \delta, \varepsilon)$ in ${ }_{H}^{H} \mathcal{Y D}$ relative to $y$ or simply the Yetter-Drinfeld datum associated to $y$ whenever there is no risk of confusion.

Definitions 1.10. [AMStu, Definitions 3.1] Let $H$ be a Hopf algebra. A cocycle for a pre-bialgebra $(R, m, u, \delta, \varepsilon)$ in ${ }_{H}^{H} \mathcal{Y} \mathcal{D}$ is a $K$-linear map

$$
\xi: R \otimes R \rightarrow H
$$

such that, for all $r, s \in R$ and $h \in H$, the following relations are satisfied:

$$
\begin{aligned}
& \sum \xi\left(h_{(1)} r \otimes h_{(2)} s\right)=\sum h_{(1)} \xi(r \otimes s) S\left(h_{(2)}\right) ; \\
& \Delta_{H} \xi=\left(m_{H} \otimes \xi\right)\left(\xi \otimes \rho_{R \otimes R}\right) \delta_{R \otimes R} \quad \text { and } \quad \varepsilon_{H} \xi=m_{K}(\varepsilon \otimes \varepsilon) ; \\
& c_{R, H}(m \otimes \xi) \delta_{R \otimes R}=\left(m_{H} \otimes m_{R}\right)\left(\xi \otimes \rho_{R \otimes R}\right) \delta_{R \otimes R} ; \\
& m_{R}\left(R \otimes m_{R}\right)=m_{R}\left(R \otimes \mu_{R}\right)\left[\left(m_{R} \otimes \xi\right) \delta_{R \otimes R} \otimes R\right] \\
& m_{H}(\xi \otimes H)\left[R \otimes\left(m_{R} \otimes \xi\right) \delta_{R \otimes R}\right]=m_{H}(\xi \otimes H)\left(R \otimes c_{H, R}\right)\left[\left(m_{R} \otimes \xi\right) \delta_{R \otimes R} \otimes R\right] \\
& \xi(R \otimes u)=\xi(u \otimes R)=\varepsilon 1_{H} .
\end{aligned}
$$

We will also say that $(R, m, u, \delta, \varepsilon)$ is a pre-bialgebra in ${ }_{H}^{H} \mathcal{Y D}$ with cocycle $\xi$.

A morphism $f:((R, m, u, \delta, \varepsilon), \xi) \rightarrow\left(\left(R^{\prime}, m^{\prime}, u^{\prime}, \delta^{\prime}, \varepsilon^{\prime}\right), \xi^{\prime}\right)$ of pre-bialgebras with a cocycle in ${ }_{H}^{H} \mathcal{Y D}$, is a morphism $f:(R, m, u, \delta, \varepsilon) \rightarrow\left(R^{\prime}, m^{\prime}, u^{\prime}, \delta^{\prime}, \varepsilon^{\prime}\right)$ of pre-bialgebras in ${ }_{H}^{H} \mathcal{Y D}$ such that

$$
\xi^{\prime} \circ(f \otimes f)=\xi \text {. }
$$

For a pre-bialgebra $(R, m, u, \delta, \varepsilon)$ in ${ }_{H}^{H} \mathcal{Y D}$ with cocycle $\xi$, we have that $(R, u, m, \xi)$ is a dual YetterDrinfeld quadruple in the sense of [AMSte, Definition 3.59]

To any pre-bialgebra $(R, m, u, \delta, \varepsilon)$ in ${ }_{H}^{H} \mathcal{Y D}$ with cocycle $\xi$ we associate (see [AMSte, Theorem 3.62]) a bialgebra $B=R \#_{\xi} H$ as follows. As a vector space it is $R \otimes H$.

The coalgebra structures are:

$$
\begin{aligned}
\Delta_{B}(r \# h) & =\sum r^{(1)} \# r_{\langle-1\rangle}^{(2)} h_{(1)} \otimes r_{\langle 0\rangle}^{(2)} \# h_{(2)}, \quad \text { where } \delta(r)=\sum r^{(1)} \otimes r^{(2)}, \\
\varepsilon_{B}(r \# h) & =\varepsilon(r) \varepsilon_{H}(h) .
\end{aligned}
$$

The algebra structures are:

$$
\begin{aligned}
m_{B}[(r \# h) \otimes(s \# k)] & =\sum \widetilde{m}^{0}\left(r \otimes{ }^{h_{(1)}} s\right) \otimes \widetilde{m}^{1}\left(r \otimes{ }^{h_{(1)}} s\right) h_{(2)} k . \\
u_{B}(1) & =u(1) \# 1_{H}
\end{aligned}
$$


where we use the notation

$$
(m \otimes \xi) \delta_{R \otimes R}(r \otimes s)=\widetilde{m}(r \otimes s)=\sum \widetilde{m}^{0}(r \otimes s) \otimes \widetilde{m}^{1}(r \otimes s)
$$

Note that the canonical injection $\sigma: H \hookrightarrow R \#_{\xi} H$ is a bialgebra homomorphism. Furthermore the map

$$
\pi: R \#_{\xi} H \rightarrow H: r \# h \longmapsto \varepsilon(r) h
$$

is an $H$-bilinear coalgebra retraction of $\sigma$.

Definitions 1.11. AMStu, Definitions 3.2] Let $H$ be a Hopf algebra, let $A$ be a bialgebra and let $\sigma: H \rightarrow A$ be an injective morphism of bialgebras having a retraction $\pi: A \rightarrow H$ (i.e. $\pi \sigma=H$ ) that is an $H$-bilinear coalgebra map. Set

$$
R=A^{C o(H)}=\left\{a \in A \mid \sum a_{(1)} \otimes \pi\left(a_{(2)}\right)=a \otimes 1_{H}\right\}
$$

Let $\tau: A \rightarrow R, \tau(a)=\sum a_{(1)} \sigma S \pi\left(a_{(2)}\right)$ (see Proposition 1.13). The map

$$
\omega: R \otimes H \rightarrow A, \omega(r \otimes h)=r \sigma(h)
$$

is an isomorphism of $K$-vector spaces, the inverse being defined by

$$
\omega^{-1}: A \rightarrow R \otimes H, \omega^{-1}(a)=\sum a_{(1)} \sigma S_{H} \pi\left(a_{(2)}\right) \otimes \pi\left(a_{(3)}\right)=\sum \tau\left(a_{(1)}\right) \otimes \pi\left(a_{(2)}\right) .
$$

Clearly $A$ defines, via $\omega$, a bialgebra structure on $R \otimes H$ that will depend on the chosen $\sigma$ and $\pi$. As shown in Scha, 6.1] and [AMSte, Theorem 3.64], $(R, m, u, \delta, \varepsilon)$ is a pre-bialgebra in ${ }_{H}^{H} \mathcal{Y D}$ with cocycle $\xi$ where

$$
\delta(r)=\sum r_{(1)} \sigma S \pi\left(r_{(2)}\right) \otimes r_{(3)}=\sum \tau\left(r_{(1)}\right) \otimes r_{(2)}, \quad \varepsilon=\varepsilon_{A \mid R},
$$

the Yetter-Drinfeld module structure of $R$ is given by

$$
{ }^{h} r=\sum \sigma\left(h_{(1)}\right) r \sigma S_{H}\left(h_{(1)}\right), \quad \rho(r)=\sum \pi\left(r_{(1)}\right) \otimes r_{(2)},
$$

the maps $u: K \rightarrow R$ and $m: R \otimes R \rightarrow R$, are defined by

$$
u=u_{A}^{\mid R}, \quad m(r \otimes s)=\sum r_{(1)} s_{(1)} \sigma S \pi\left(r_{(2)} s_{(2)}\right)=\tau\left(r \cdot{ }_{A} s\right)
$$

and the cocycle $\xi: R \otimes R \rightarrow H$ is the map defined by setting

$$
\xi(r \otimes s)=\pi(r \cdot A s) .
$$

This pre-bialgebra will be called the pre-bialgebra in ${ }_{H}^{H} \mathcal{Y D}$ associated to $(A, \pi, \sigma)$. Moreover $\xi$ will be called the cocycle corresponding to $(R, m, u, \delta, \varepsilon)$.

Then (cf. Scha, 6.1]) $\omega: R \#_{\xi} H \rightarrow A$ is a bialgebra isomorphism.

REMARK 1.12. Note that, starting from a pre-bialgebra $(R, m, u, \delta, \varepsilon)$ in ${ }_{H}^{H} \mathcal{Y D}$ with cocycle $\xi$, if we consider the maps

$$
\sigma: H \hookrightarrow R \#_{\xi} H \quad \text { and } \quad \pi: R \#_{\xi} H \rightarrow H
$$

as in Definitions 1.10, then the pre-bialgebra in ${ }_{H}^{H} \mathcal{Y D}$ associated to $\left(R \#_{\xi} H, \pi, \sigma\right)$ is exactly $(R, m, u, \delta, \varepsilon)$ and the corresponding cocycle is exactly $\xi$.

Proposition 1.13. AMStu, Proposition 3.4] Let $H$ be a Hopf algebra with antipode $S$, let $A$ be a bialgebra and let $\sigma: H \rightarrow A$ be an injective morphism of bialgebras having a retraction $\pi: A \rightarrow H$ (i.e. $\pi \sigma=H)$ that is an $H$-bilinear coalgebra map. Let $(R, m, u, \delta, \varepsilon)$ be the pre-bialgebra in ${ }_{H}^{H} \mathcal{Y D}$ associated to $(A, \pi, \sigma)$.

Then the map $\tau$ of Definitions 1.11 is a surjective coalgebra homomorphism. Moreover

$$
\begin{aligned}
\tau[a \sigma(h)] & =\tau(a) \varepsilon_{H}(h), & \tau[\sigma(h) a] & ={ }^{h} \tau(a), \\
r \cdot \cdot_{R} s & =\tau\left(r \cdot \cdot_{A} s\right), & \tau(a) \cdot \cdot_{R} \tau(b) & =\tau\left[\tau(a) \cdot{ }_{A} b\right],
\end{aligned}
$$

where $a \in A, h \in H$ and $r, s \in R$. 
1.14. Let $H$ be a Hopf algebra and let $\chi \in H^{*}$ be a character. Let $\left(M, \rho_{M}\right)$ be a left $H$-comodule and $\left(N, \rho_{N}\right)$ be a right $H$-comodule. In the sequel we will use the well known $K$-linear automorphisms $\varphi_{M}: M \rightarrow M$ and $\psi_{N}: N \rightarrow N$ defined by

$$
\varphi_{M}(m)=(m<\chi)=\sum \chi\left(m_{\langle-1\rangle}\right) m_{\langle 0\rangle} \quad \text { and } \quad \psi_{N}(n)=(\chi \rightarrow n)=\sum n_{\langle 0\rangle} \chi\left(n_{\langle 1\rangle}\right)
$$

Recall that $\varphi_{M}$ and $\psi_{N}$ are (co)algebra automorphisms whenever $M$ and $N$ are $H$-comodule (co)algebras.

Proposition 1.15. Let $H$ be a Hopf algebra over a field $K$. Let $i=1,2$. Let $A^{i}$ be a bialgebra and let $\sigma^{i}: H \rightarrow A^{i}$ be an injective morphism of bialgebras having a retraction $\pi^{i}: A^{i} \rightarrow H$ (i.e. $\left.\pi^{i} \sigma^{i}=H\right)$ that is an H-bilinear coalgebra map. Denote by $\left(R^{i}, m^{i}, u^{i}, \delta^{i}, \varepsilon^{i}\right)$ the pre-bialgebra in ${ }_{H}^{H} \mathcal{Y} \mathcal{D}$ associated to $\left(A^{i}, \pi^{i}, \sigma^{i}\right)$ with corresponding cocycle $\xi^{i}$. Set

$$
\tau^{i}: A^{i} \rightarrow R^{i}: \tau^{i}(a)=a_{(1)} \sigma^{i} S \pi^{i}\left(a_{(2)}\right) \quad \text { and } \quad \omega^{i}: R^{i} \# \xi_{\xi^{i}} H \rightarrow A^{i}, \omega^{i}(u \otimes h)=u \cdot A^{2} \sigma^{i}(h) .
$$

The assignments

$$
\Phi \mapsto \tau^{2} \Phi_{\mid R^{1}} \quad \text { and } \quad \phi \mapsto \omega^{2}(\phi \otimes H)\left(\omega^{1}\right)^{-1}
$$

define a bijective correspondence between the following data:

(i) (iso)morphisms of bialgebras $\Phi: A^{1} \rightarrow A^{2}$ such that $\Phi \circ \sigma^{1}=\sigma^{2}$ and $\pi^{2} \circ \Phi=\pi^{1}$.

(ii) (iso)morphisms $\phi:\left(\left(R^{1}, m^{1}, u^{1}, \delta^{1}, \varepsilon^{1}\right), \xi^{1}\right) \rightarrow\left(\left(R^{2}, m^{2}, u^{2}, \delta^{2}, \varepsilon^{2}\right), \xi^{2}\right)$ of pre-bialgebras with a cocycle in ${ }_{H}^{H} \mathcal{Y} \mathcal{D}$.

Proof. It is straightforward.

Lemma 1.16. Let $H$ be a Hopf algebra over a field $K$. Let $A$ be a bialgebra and let $\sigma: H \rightarrow A$ be an injective morphism of bialgebras having a retraction $\pi: A \rightarrow H$ (i.e. $\left.\pi \sigma=\operatorname{Id}_{H}\right)$ that is an $H$-bilinear coalgebra map. Let $(R, m, u, \delta, \varepsilon)$ be the pre-bialgebra in ${ }_{H}^{H} \mathcal{Y} \mathcal{D}$ associated to $(A, \pi, \sigma)$ and let $\xi$ be the corresponding cocycle. Assume that $R$ is a connected coalgebra.

Then the following assertions are equivalent.

(i) $H$ is the coradical of $A$.

(ii) $H$ is cosemisimple.

Proof. $(i) \Rightarrow($ ii $)$ It is trivial.

$($ ii $) \Rightarrow(i)$ By Definitions 1.11, the morphism $\omega: R \#_{\xi} H \rightarrow A, \omega(r \otimes h)=r \sigma(h)$ is a bialgebra isomorphism. Since $R$ is connected, by AMStu, Theorem 3.9], we have $\left(R \#_{\xi} H\right)_{0}=K \otimes H_{0}=$ $K \otimes H$. Through $\omega$ we get $A_{0}=H$.

\section{THE COCYCLE}

Definition 2.1. Let $C$ be a $K$-coalgebra, let $s \in \mathbb{N}$ and let $d_{0}, d_{1}, \ldots, d_{s} \in C$. Recall that $\left(d_{i}\right)_{0 \leq i \leq s}$ is called a divided power sequence of elements in $C$ whenever

$$
\Delta\left(d_{n}\right)=\sum_{t=0}^{n} d_{t} \otimes d_{n-t}
$$

for any $0 \leq n \leq s$.

Notation 2.2. Let $H$ be a Hopf algebra and let $(R, m, u, \delta, \varepsilon)$ be a $N$-dimensional pre-bialgebra in ${ }_{H}^{H} \mathcal{Y D}$ with cocycle $\xi$ in the sense of Definitions 1.3 and 1.10. Assume that $R$ is a thin coalgebra where $P(R)=K y$. Let $g \in H$ and $\chi \in H^{*}$ be such that $(H, g, \chi)$ is the Yetter-Drinfeld datum associated to $y$ (see Definition 1.9) and let $q=\chi(\mathrm{g})$.

From now on, we fix a basis of $R$ consisting of a divided power sequence of non-zero elements in $R$

$$
d_{0}=1_{R}, d_{1}=y, \ldots, d_{N-1}
$$


such that

$$
\begin{aligned}
g d_{n} & =q^{n} d_{n}, \text { for any } 0 \leq n \leq N-1, \\
y d_{n-1} & =(n)_{q} d_{n}, \text { for any } 1 \leq n \leq N-1, \\
h d_{n} & =\chi^{n}(h) d_{n}, \text { for any } 0 \leq n \leq N-1 .
\end{aligned}
$$

Such a basis exists in view of [AMStu, Lemmata 2.9 and 2.15]. From now on, we will also use the following notation

$$
Y:=y \otimes 1_{H}, \quad \Gamma:=1_{R} \otimes g,
$$

and we will denote by $\mathcal{B}(H)$ a basis for $H$. For $N$ even we set

$$
x:=\xi\left(d_{1} \otimes d_{N / 2-1}\right), \quad X:=(N / 2-1)_{q} ! \cdot\left(1_{R} \otimes x\right) .
$$

For every $n \in \mathbb{N}$, we will denote by $Y^{n}$ and $X^{n}$ the $n$-th power of $Y$ and $X$ in $R \#_{\xi} H$ respectively.

We recall from AMStu] some results that will be needed in the sequel.

Lemma 2.3. Keep the assumptions and notations of 2.9.

i) AMStu, formula (24) in Lemma 3.10] Let $0 \leq a, b \leq N-1$. Then,

$$
\chi^{a+b}(h) \xi\left(d_{a} \otimes d_{b}\right)=\sum h_{(1)} \xi\left(d_{a} \otimes d_{b}\right) S h_{(2)}, \text { for every } h \in H .
$$

ii) AMStu, formula (31) in Theorem 3.11] If $0 \leq a \leq N-1$, we have

$$
\chi^{c}\left[\xi\left(d_{1} \otimes d_{a}\right)\right]=0, \text { for any } c \in \mathbb{N} .
$$

iii) AMStu, formula (32) in Theorem 3.11] If $0 \leq a, b \leq N$ and $b \leq N-a$, we have

$$
\begin{aligned}
& \rho\left(d_{a} d_{b}\right)=\sum\left(d_{a}\right)_{\langle-1\rangle}\left(d_{b}\right)_{\langle-1\rangle} \otimes\left(d_{a}\right)_{\langle 0\rangle}\left(d_{b}\right)_{\langle 0\rangle}+ \\
& +\sum_{\substack{0 \leq i \leq a, 0 \leq j \leq b \\
0<i+j<a+b}}\left[\begin{array}{c}
q^{(b-j) i} \xi\left(d_{a-i} \otimes d_{b-j}\right)\left(d_{i}\right)_{\langle-1\rangle}\left(d_{j}\right)_{\langle-1\rangle} \otimes\left(d_{i}\right)_{\langle 0\rangle}\left(d_{j}\right)_{\langle 0\rangle}+ \\
-q^{j(a-i)}\left(d_{i} d_{j}\right)_{\langle-1\rangle} \xi\left(d_{a-i} \otimes d_{b-j}\right) \otimes\left(d_{i} d_{j}\right)_{\langle 0\rangle}
\end{array}\right] .
\end{aligned}
$$

iv) AMStu, formula (36)] For every $0 \leq a, b \leq N-1$

$$
\left(m_{R} \otimes \xi\right) \delta_{R \otimes R}\left(d_{a} \otimes d_{b}\right)=\sum_{0 \leq i \leq a, 0 \leq j \leq b} q^{j(a-i)}\left(d_{i} d_{j}\right) \otimes \xi\left(d_{a-i} \otimes d_{b-j}\right) .
$$

v) AMStu, formula (40)] For every $0 \leq a, b \leq N-1, c \geq 0$

$$
\begin{gathered}
\varphi_{H}^{c}\left[\xi\left(d_{a} \otimes d_{b}\right)\right]=q^{c(a+b)} \xi\left(d_{a} \otimes d_{b}\right) \\
\psi_{H}^{c}\left[\xi\left(d_{a} \otimes d_{b}\right)\right]=\xi\left(d_{a} \otimes d_{b}\right) .
\end{gathered}
$$

vi) AMStu, formula (44) in Lemma 3.16] For any $a, b \in \mathbb{N}$ such that $0 \leq a, b \leq N-1$, we have:

$$
\xi\left(d_{a} \otimes d_{b}\right)=0 \text { unless } a+b=0, \frac{N}{2}, N, \frac{3 N}{2}
$$

whenever this makes sense.

TheOREM 2.4. AMStu, Theorem 3.14]Keep the assumptions and notations of 2.9. Then:

1) $R$ is an associative algebra over $K$ spanned by $y$.

2) $o(q)=N$.

3) $y^{n}=(n)_{q} ! d_{n}$, for every $0 \leq n \leq N-1$ and $y^{N}=0$.

4) $\left(y^{i}\right)_{0 \leq i \leq N-1}$ is a basis for $R$.

5) $R=\bar{R}_{q}(H, g, \chi)$ is a quantum line, whenever $m$ is left $H$-colinear.

Next aim is to study the behavior of $\xi$. 
Proposition 2.5. Keep the assumptions and notations of 2.9. Let $0 \leq a, b, c \leq N-1$. We have

$$
\begin{aligned}
& \sum_{0 \leq i \leq b, 0 \leq j \leq c} q^{j(b-i)} \xi\left(d_{a} \otimes d_{i} d_{j}\right) \xi\left(d_{b-i} \otimes d_{c-j}\right) \\
= & \sum_{0 \leq i \leq a, 0 \leq j \leq b} q^{(j+c)(a-i)+c(b-j)} \xi\left(d_{i} d_{j} \otimes d_{c}\right) \xi\left(d_{a-i} \otimes d_{b-j}\right) .
\end{aligned}
$$

Moreover we obtain

$$
\begin{aligned}
& \xi\left(d_{a} \otimes d_{1} d_{c}\right)-\xi\left(d_{a} d_{1} \otimes d_{c}\right) \\
= & \sum_{0 \leq i<a} q^{c(a-i)+c} \xi\left(d_{i} \otimes d_{c}\right) \xi\left(d_{a-i} \otimes d_{1}\right)-\sum_{0 \leq j<c} q^{j} \xi\left(d_{a} \otimes d_{j}\right) \xi\left(d_{1} \otimes d_{c-j}\right)
\end{aligned}
$$

Proof. We evaluate the first term of (13) on $d_{a} \otimes d_{b} \otimes d_{c}$.

$$
\begin{array}{ll} 
& m_{H}(\xi \otimes H)\left[R \otimes\left(m_{R} \otimes \xi\right) \delta_{R \otimes R}\right]\left(d_{a} \otimes d_{b} \otimes d_{c}\right) \\
= & m_{H}(\xi \otimes H)\left[d_{a} \otimes\left(m_{R} \otimes \xi\right) \delta_{R \otimes R}\left(d_{b} \otimes d_{c}\right)\right] \\
\text { 201 } & \sum_{0 \leq i \leq b, 0 \leq j \leq c} q^{j(b-i)} \xi\left(d_{a} \otimes d_{i} d_{j}\right) \xi\left(d_{b-i} \otimes d_{c-j}\right)
\end{array}
$$

We evaluate the second term of (13) on $d_{a} \otimes d_{b} \otimes d_{c}$.

$$
\begin{array}{ll} 
& m_{H}(\xi \otimes H)\left(R \otimes c_{H, R}\right)\left[\left(m_{R} \otimes \xi\right) \delta_{R \otimes R} \otimes R\right]\left(d_{a} \otimes d_{b} \otimes d_{c}\right) \\
= & m_{H}(\xi \otimes H)\left(R \otimes c_{H, R}\right)\left[\left(m_{R} \otimes \xi\right) \delta_{R \otimes R}\left(d_{a} \otimes d_{b}\right) \otimes d_{c}\right] \\
\text { 20] } & \sum_{0 \leq i \leq a, 0 \leq j \leq b} q^{j(a-i)} m_{H}(\xi \otimes H)\left\{d_{i} d_{j} \otimes c_{H, R}\left[\xi\left(d_{a-i} \otimes d_{b-j}\right) \otimes d_{c}\right]\right\}
\end{array}
$$

Since $c_{H, R}(h \otimes r)=\sum h_{1} r \otimes h_{2}$, we have

$$
c_{H, R}\left(h \otimes d_{c}\right)=\sum h_{1} d_{c} \otimes h_{2}=\sum \chi^{c}\left(h_{1}\right) d_{c} \otimes h_{2}=d_{c} \otimes \varphi_{H}^{c}(h),
$$

that is $c_{H, R}\left(h \otimes d_{c}\right)=d_{c} \otimes \varphi_{H}^{c}(h)$. By (21), we get

$$
c_{H, R}\left[\xi\left(d_{a-i} \otimes d_{b-j}\right) \otimes d_{c}\right]=d_{c} \otimes \varphi_{H}^{c}\left[\xi\left(d_{a-i} \otimes d_{b-j}\right)\right]=q^{c[a+b-(i+j)]} d_{c} \otimes \xi\left(d_{a-i} \otimes d_{b-j}\right) .
$$

Therefore we obtain

$$
\begin{aligned}
& m_{H}(\xi \otimes H)\left(R \otimes c_{H, R}\right)\left[\left(m_{R} \otimes \xi\right) \delta_{R \otimes R} \otimes R\right]\left(d_{a} \otimes d_{b} \otimes d_{c}\right) \\
= & \sum_{0 \leq i \leq a, 0 \leq j \leq b} q^{j(a-i)} m_{H}(\xi \otimes H)\left\{d_{i} d_{j} \otimes c_{H, R}\left[\xi\left(d_{a-i} \otimes d_{b-j}\right) \otimes d_{c}\right]\right\} \\
20 & \sum_{0 \leq i \leq a, 0 \leq j \leq b} q^{(j+c)(a-i)+c(b-j)} \xi\left(d_{i} d_{j} \otimes d_{c}\right) \xi\left(d_{a-i} \otimes d_{b-j}\right)
\end{aligned}
$$

Finally by (13), we have (24). For $b=1$ we obtain

$$
\begin{aligned}
& \sum_{0 \leq j \leq c} q^{j} \xi\left(d_{a} \otimes d_{j}\right) \xi\left(d_{1} \otimes d_{c-j}\right)+\sum_{0 \leq j \leq c} \xi\left(d_{a} \otimes d_{1} d_{j}\right) \xi\left(1_{R} \otimes d_{c-j}\right) \\
= & \sum_{0 \leq i \leq a} q^{c(a-i)+c} \xi\left(d_{i} \otimes d_{c}\right) \xi\left(d_{a-i} \otimes d_{1}\right)+\sum_{0 \leq i \leq a} q^{(1+c)(a-i)} \xi\left(d_{i} d_{1} \otimes d_{c}\right) \xi\left(d_{a-i} \otimes 1_{R}\right) .
\end{aligned}
$$

By (14) we get

$$
\begin{gathered}
\sum_{0 \leq j \leq c} q^{j} \xi\left(d_{a} \otimes d_{j}\right) \xi\left(d_{1} \otimes d_{c-j}\right)+\xi\left(d_{a} \otimes d_{1} d_{c}\right) \\
=\sum_{0 \leq i \leq a} q^{c(a-i)+c} \xi\left(d_{i} \otimes d_{c}\right) \xi\left(d_{a-i} \otimes d_{1}\right)+\xi\left(d_{a} d_{1} \otimes d_{c}\right)
\end{gathered}
$$

Finally we have that

$$
\begin{aligned}
& \xi\left(d_{a} \otimes d_{1} d_{c}\right)-\xi\left(d_{a} d_{1} \otimes d_{c}\right) \\
= & \sum_{0 \leq i \leq a} q^{c(a-i)+c} \xi\left(d_{i} \otimes d_{c}\right) \xi\left(d_{a-i} \otimes d_{1}\right)-\sum_{0 \leq j \leq c} q^{j} \xi\left(d_{a} \otimes d_{j}\right) \xi\left(d_{1} \otimes d_{c-j}\right) \\
= & \sum_{0 \leq i<a} q^{c(a-i)+c} \xi\left(d_{i} \otimes d_{c}\right) \xi\left(d_{a-i} \otimes d_{1}\right)-\sum_{0 \leq j<c} q^{j} \xi\left(d_{a} \otimes d_{j}\right) \xi\left(d_{1} \otimes d_{c-j}\right) .
\end{aligned}
$$

Statement 7) in the following Lemma 2.6 has already been proved in [AMStu, Lemma 3.26]. 
LEMmA 2.6. Keep the assumptions and notations of 2.7. Assume that $N$ is even and let $x=$ $\xi\left(d_{1} \otimes d_{N / 2-1}\right)$. Then we have

1) $\Delta_{H}(x)=g^{N / 2} \otimes x+x \otimes 1_{H}$.

2) $\chi^{c}(x)=0$, for any $c \in \mathbb{N}$.

$\left.2^{\prime}\right) \varphi_{H}^{c}(x)=(-1)^{c} x$ and $\psi_{H}^{c}(x)=x$ for any $c \in \mathbb{N}$.

3) $\chi^{N / 2}(h) x=\sum h_{(1)} x S\left(h_{(2)}\right)$, for any $h \in H$.

4) $x g+g x=0$.

5) $N / 2=1 \Longrightarrow x=0$ and $N / 2$ is odd $\Longrightarrow x^{2}=0$.

6) $N / 2$ even and $H$ finite dimensional $\Longrightarrow x=0$.

7) $H$ cosemisimple $\Longrightarrow x=0$.

Proof. 1) By AMStu, Lemma 3.25], for any $1 \leq b \leq N / 2$, we have

$$
\Delta_{H} \xi\left(d_{1} \otimes d_{b}\right)=g^{1+b} \otimes \xi\left(d_{1} \otimes d_{b}\right)+\xi\left(d_{1} \otimes d_{b}\right) \otimes 1_{H} .
$$

In particular, if $N \geq 4$, we can apply this formula for $b=N / 2-1$ and obtain $\Delta_{H}(x)=g^{N / 2} \otimes$ $x+x \otimes 1_{H}$. This equality still holds whenever $N=2$ as in this case $x=\xi\left(d_{1} \otimes d_{N / 2-1}\right)=$ $\xi\left(d_{1} \otimes d_{0}\right) \stackrel{14}{=} \varepsilon\left(d_{1}\right)=0$. Hence we obtained 1) and the first part of 5).

2) Let $c \in \mathbb{N}$. By (18), for every $0 \leq a \leq N-1$, we have $\chi^{c}\left[\xi\left(d_{1} \otimes d_{a}\right)\right]=0$ and hence $\chi^{c}(x)=0$.

$\left.2^{\prime}\right)$ By formula 21 and 22, we have

$$
\varphi_{H}^{c}\left[\xi\left(d_{a} \otimes d_{b}\right)\right]=q^{c(a+b)} \xi\left(d_{a} \otimes d_{b}\right), \quad \psi_{H}^{c}\left[\xi\left(d_{a} \otimes d_{b}\right)\right]=\xi\left(d_{a} \otimes d_{b}\right)
$$

so that $\varphi_{H}^{c}(x)=\left(q^{N / 2}\right)^{c} x=(-1)^{c} x$ and $\psi_{H}^{c}(x)=x$.

3) By (17), for any $0 \leq a, b \leq N-1$ and for any $h \in H$, we have

$$
\chi^{a+b}(h) \xi\left(d_{a} \otimes d_{b}\right)=\sum h_{(1)} \xi\left(d_{a} \otimes d_{b}\right) S\left(h_{(2)}\right) .
$$

In particular, for $(a, b)=(1, N / 2-1)$, we get the required formula.

4) If $h=g$, from 3) we obtain $q^{N / 2} x=g x g^{-1}$ that is $x g+g x=0$. In particular

$$
g^{N / 2} x=(-1)^{N / 2} x g^{N / 2} .
$$

5) We have

$$
0 \stackrel{2)}{=} \chi^{N / 2}(x) x \stackrel{3)}{=} \sum x_{(1)} x S\left(x_{(2)}\right) .
$$

From 1), we get $S(x)=-g^{-N / 2} x$ so that

$$
\begin{aligned}
\sum x_{(1)} x S\left(x_{(2)}\right) \stackrel{1)}{=} g^{N / 2} x S(x)+x x S\left(1_{H}\right)=g^{N / 2} x\left(-g^{-N / 2} x\right)+x^{2} & = \\
\stackrel{27}{=}(-1)^{N / 2} x g^{N / 2}\left(-g^{-N / 2} x\right)+x^{2} & =\left[-(-1)^{N / 2}+1\right] x^{2}
\end{aligned}
$$

Thus from (28), we obtain $\left[-(-1)^{N / 2}+1\right] x^{2}=0$. Now, if $N / 2$ is odd we get $2 x^{2}=0$ and hence $x^{2}=0(\operatorname{char}(K)=0)$.

6 ) If $N / 2$ is even, then, from (27), we infer $x g^{N / 2}=g^{N / 2} x$. Now by 1$), \Delta_{H}(x)=g^{N / 2} \otimes x+x \otimes 1_{H}$. Since $H$ is finite dimensional there exists $\lambda(x) \in K$ such that $x=\lambda(x)\left(1_{H}-g^{N / 2}\right)$ (see e.g. [AMStu, Theorem 0.1]) so that $x g=g x$. From 4) we also have $x g+g x=0$ so that $x g=0$ and hence $x=0$.

7) Assume now that $H$ is cosemisimple and let $\lambda \in H^{*}$ be a total integral. Then, regardless to the parity of $N / 2$, by applying $H \otimes \lambda$ to $\Delta_{H}(x) \stackrel{1)}{=} g^{N / 2} \otimes x+x \otimes 1_{H}$ we get $x=\lambda(x)\left(1_{H}-g^{N / 2}\right)$ so that, as above, from $x g+g x=0$ we obtain $x g=0$ and hence $x=0$.

Proposition 2.7. AMStu, Proposition 3.21] Take the hypothesis and notations of 2.2. If $N$ is odd we have

$$
Y^{a}= \begin{cases}y^{a} \otimes 1_{H} & \text { for } 0 \leq a \leq N-1 \\ 1_{R} \otimes \xi\left(y \otimes y^{N-1}\right) & \text { for } a=N .\end{cases}
$$


If $N$ is even, we have

$$
Y^{a}= \begin{cases}y^{a} \otimes 1_{H} & \text { for } 0 \leq a \leq N / 2-1 \\
\left(\begin{array}{c}
a \\
N / 2
\end{array}\right)_{q} Y^{a-N / 2} \cdot{ }_{R \#_{\xi} H} X+y^{a} \otimes 1_{H} & \text { for } N / 2 \leq a \leq N-1 \\
1_{R} \otimes \xi\left(y \otimes y^{N-1}\right)+\left(\begin{array}{c}
N-1 \\
N / 2
\end{array}\right)_{q} X^{2} & \text { for } a=N\end{cases}
$$

where $X=(N / 2-1)_{q} ! \cdot\left(1_{R} \otimes x\right)$.

Proposition 2.8. Keep the assumptions and notations of 2.9.

1) Assume that $N$ is even and let $x=\xi\left(d_{1} \otimes d_{N / 2-1}\right)$. Then, we have:

$$
\xi\left(y \otimes y^{N / 2-1}\right)=\xi\left(y^{2} \otimes y^{N / 2-2}\right)=\cdots=\xi\left(y^{N / 2-1} \otimes y\right)=(N / 2-1)_{q} ! x
$$

2) If $x^{2}=0$, then we have

$$
\xi\left(y \otimes y^{N-1}\right)=\xi\left(y^{2} \otimes y^{N-2}\right)=\cdots=\xi\left(y^{N-1} \otimes y\right) .
$$

Moreover in $R \#_{\xi} H$ we have

$$
Y^{N}=1_{R} \otimes \xi\left(y \otimes y^{N-1}\right)
$$

3) If $x=0$, then we have

$$
\xi\left(y \otimes y^{3 N / 2-1}\right)=\xi\left(y^{2} \otimes y^{3 N / 2-2}\right)=\cdots=\xi\left(y^{3 N / 2-1} \otimes y\right)=0 .
$$

Proof. Let $0 \leq a, b \leq N-1$. Then, from (23), we infer that

$$
\xi\left(y^{a} \otimes y^{b}\right)=0 \text { unless } a+b=0, \frac{N}{2}, N, \frac{3 N}{2},
$$

whenever they make sense.

By Proposition 2.5 we have (25).

1) Let us consider the case $1 \leq a, 1 \leq c$ and $a+c+1 \leq N / 2+1$

Then for $0 \leq i \leq a-1$ we have $i+c \leq a-1+c \leq N / 2-1$ so that, by (23), we get $\xi\left(d_{i} \otimes d_{c}\right)=0$.

Analogously for $0 \leq j \leq c-1$ we have $j+a \leq c-1+a \leq N / 2-1$ so that $\xi\left(d_{a} \otimes d_{j}\right)=0$. Hence if $1 \leq a, 1 \leq c$ and $a+c+1 \leq N / 2+1$, by (25), we obtain $\xi\left(d_{a} \otimes d_{1} d_{c}\right)=\xi\left(d_{a} d_{1} \otimes d_{c}\right)$. Since, in view of Theorem 2.4. $y^{n}=(n)_{!} ! d_{n}$, for every $0 \leq n \leq N-1$, we deduce that $\xi\left(y^{a} \otimes y^{1+c}\right)=\xi\left(y^{a+1} \otimes y^{c}\right)$. It is now clear that (29) holds.

2) Assume $x^{2}=0$. Let us prove that for any $1 \leq a, 1 \leq c$ and $a+c+1=N$ one has $\xi\left(d_{a} \otimes d_{1} d_{c}\right)=\xi\left(d_{a} d_{1} \otimes d_{c}\right)$. As above we will apply (25).

$$
\begin{aligned}
\xi\left(d_{a} \otimes d_{1} d_{c}\right)-\xi\left(d_{a} d_{1} \otimes d_{c}\right) & \\
=\sum_{0 \leq i<a} q^{c(a-i)+c} \xi\left(d_{i} \otimes d_{c}\right) \xi( & \left.d_{a-i} \otimes d_{1}\right)-\sum_{0 \leq j<c} q^{j} \xi\left(d_{a} \otimes d_{j}\right) \xi\left(d_{1} \otimes d_{c-j}\right) \\
\sum_{0 \leq j<c} q^{j} \xi\left(d_{a} \otimes d_{j}\right) \xi\left(d_{1} \otimes d_{c-j}\right) & =q^{c-N / 2+1} \xi\left(d_{a} \otimes d_{c-N / 2+1}\right) \xi\left(d_{1} \otimes d_{N / 2-1}\right) \\
& =q^{N / 2-a} \xi\left(d_{a} \otimes d_{N / 2-a}\right) \xi\left(d_{1} \otimes d_{N / 2-1}\right) \\
\sum_{0 \leq i<a} q^{c(a-i)+c} \xi\left(d_{i} \otimes d_{c}\right) \xi\left(d_{a-i} \otimes d_{1}\right) & =q^{c(N / 2-1)+c}\left[\xi\left(d_{N / 2-c} \otimes d_{c}\right) \xi\left(d_{N / 2-1} \otimes d_{1}\right)\right] \\
q^{N / 2-a} & =q^{c(N / 2-1)+c} \\
q^{c(N / 2-1)+c} & =\left(q^{N / 2}\right)^{c}=(-1)^{c} \\
q^{N / 2-a} & =-q^{-a}=-q^{c+1-N}
\end{aligned}
$$

Let us consider the case $1 \leq a, 1 \leq c$ and $a+c+1=N$.

Assume $N$ odd. Then for $0 \leq i \leq a$ we have $i+c \leq a+c=N-1$ so that, by (23), $\xi\left(d_{i} \otimes d_{c}\right)=0$ always. Analogously $\xi\left(d_{a} \otimes d_{j}\right)=0$ for any $j$ such that $0 \leq j \leq c$. Thus, in the case $N$ is odd, one has $\xi\left(d_{a} \otimes d_{1} d_{c}\right)=\xi\left(d_{a} d_{1} \otimes d_{c}\right)$. 
Assume $N$ even. Then for $0 \leq i \leq a$ we have $i+c \leq a+c=N-1$ so that $\xi\left(d_{i} \otimes d_{c}\right)=0$ unless $i+c=N / 2$. In this case we obtain $a-i=a-N / 2+c=N-1-N / 2=N / 2-1$. Since, by (29), we have

$$
\xi\left(d_{a} \otimes d_{b}\right)=\frac{1}{(a)_{q} !(b)_{q} !} \xi\left(y^{a} \otimes y^{b}\right)=\frac{(N / 2-1)_{q} !}{(a)_{q} !(N / 2-a)_{q} !} x=\frac{1}{(N / 2)_{q}}\left(\begin{array}{c}
N / 2 \\
a
\end{array}\right) x
$$

for any $1 \leq a, 1 \leq b$ such that $a+b=N / 2$, we get

$$
\begin{aligned}
& \sum_{0 \leq i<a} q^{c(a-i)+c} \xi\left(d_{i} \otimes d_{c}\right) \xi\left(d_{a-i} \otimes d_{1}\right)=q^{c(N / 2-1)+c}\left[\xi\left(d_{N / 2-c} \otimes d_{c}\right) \xi\left(d_{N / 2-1} \otimes d_{1}\right)\right] \\
= & q^{c N / 2} \frac{1}{(N / 2)_{q}}\left(\begin{array}{c}
N / 2 \\
c
\end{array}\right)_{q} x \frac{1}{(N / 2)_{q}}\left(\begin{array}{c}
N / 2 \\
1
\end{array}\right)_{q} x \\
= & q^{c N / 2}\left[\frac{1}{(N / 2)_{q}}\right]^{2}\left(\begin{array}{c}
N / 2 \\
c
\end{array}\right)_{q}\left(\begin{array}{c}
N / 2 \\
1
\end{array}\right)_{q} x^{2}=0
\end{aligned}
$$

In an analogous way we get

$$
\sum_{0 \leq j<c} q^{j} \cdot\left[\xi\left(d_{a} \otimes d_{j}\right) \xi\left(d_{1} \otimes d_{c-j}\right)\right]=0
$$

so that for $1 \leq a, 1 \leq c$ and $a+c+1=N$, from (25), we deduce that $\xi\left(d_{a} \otimes d_{1} d_{c}\right)=\xi\left(d_{a} d_{1} \otimes d_{c}\right)$.

Therefore for any $1 \leq a, 1 \leq c$ and $a+c+1=N$ one has $\xi\left(d_{a} \otimes d_{1} d_{c}\right)=\xi\left(d_{a} d_{1} \otimes d_{c}\right)$ both in the even and in the odd case. Since $y^{n}=(n)_{q} ! d_{n}$, for every $0 \leq n \leq N-1$, then we obtain $\xi\left(y^{a} \otimes y^{1+c}\right)=\xi\left(y^{a+1} \otimes y^{c}\right)$. Therefore, we have $\xi\left(y \otimes y^{N-1}\right)=\xi\left(y^{2} \otimes y^{N-2}\right)=\cdots=\xi\left(y^{N-1} \otimes y\right)$.

Let us check that $Y^{N}=1_{R} \otimes \xi\left(y \otimes y^{N-1}\right)$ regardless the parity of $N$.

By Proposition 2.7, we have

$$
Y^{N}= \begin{cases}1_{R} \otimes \xi\left(y \otimes y^{N-1}\right) & \text { if } N \text { is odd } \\
1_{R} \otimes \xi\left(y \otimes y^{N-1}\right)+\left(\begin{array}{l}
N-1 \\
N / 2
\end{array}\right)_{q} X^{2} & \text { if } N \text { is even }\end{cases}
$$

where, if $N$ is even, by definition $X=(N / 2-1)_{q} ! 1_{R} \otimes x$. Since $x^{2}=0$, we obtain $X^{2}=$ $\left[(N / 2-1)_{q} !\right]^{2} 1_{R} \otimes x^{2}=0$ and hence we get 31 regardless to the parity of $N$.

3) Assume $x=0$ and let us prove that

$$
\xi\left(y^{a} \otimes y^{b}\right)=0 \text { for } a+b=\frac{3 N}{2}, 1 \leq a, b \leq N-1 .
$$

Let $u, v \in \mathbb{N}$ be such that $1 \leq u, v \leq N-2$, and $u+v+1=3 N / 2$. Then for any $0 \leq i<u$ we have $1 \leq i+v<u+v=3 N / 2-1$ so that $\xi\left(d_{i} \otimes d_{\nu}\right)=\frac{1}{(i)_{q} !(\nu)_{q} !} \xi\left(y^{i} \otimes y^{\nu}\right)=0$ unless $i+v=N$. In this case, since

$$
(i+v)+(u-i+1)=v+u+1=3 N / 2
$$

we have $u-i+1=3 N / 2-N=N / 2$ and hence $\xi\left(d_{u-i} \otimes d_{1}\right)=\frac{1}{(u-i)_{q} !} \xi\left(y^{u-i} \otimes y\right) \stackrel{29}{=} 0$.

In conclusion, for any $1 \leq u, v \leq N-2$, such that $u+v+1=3 N / 2$, we have

$$
\xi\left(d_{i} \otimes d_{\nu}\right) \xi\left(d_{u-i} \otimes d_{1}\right)=0, \text { for } 0 \leq i<u \text {. }
$$

Similarly, for any $1 \leq u, v \leq N-2$, such that $u+v+1=3 N / 2$, we have

$$
\xi\left(d_{u} \otimes d_{j}\right) \xi\left(d_{1} \otimes d_{\nu-j}\right)=0, \text { for } 0 \leq j<v .
$$

Therefore, by 25, for any $1 \leq u, v \leq N-2$, such that $u+v+1=3 N / 2$, we obtain

$$
\begin{aligned}
& \xi\left(d_{u} \otimes d_{1} d_{\nu}\right)-\xi\left(d_{u} d_{1} \otimes d_{\nu}\right) \\
= & \sum_{0 \leq i<u} q^{\nu(u-i)+\nu} \xi\left(d_{i} \otimes d_{\nu}\right) \xi\left(d_{u-i} \otimes d_{1}\right)-\sum_{0 \leq j<\nu} q^{j} \xi\left(d_{u} \otimes d_{j}\right) \xi\left(d_{1} \otimes d_{\nu-j}\right)=0 .
\end{aligned}
$$

From this we infer that, for any $1 \leq u, v \leq N-2$, such that $u+v+1=3 N / 2$, we have $\xi\left(y^{u} \otimes y^{1+\nu}\right)=\xi\left(y^{u+1} \otimes y^{\nu}\right)$. Thus, since $3 N / 2-1 \geq N$, we have $y^{3 N / 2-1}=0$ and hence we get

$$
0=\xi\left(y \otimes y^{3 N / 2-1}\right)=\xi\left(y^{2} \otimes y^{3 N / 2-2}\right)=\cdots=\xi\left(y^{N-1} \otimes y^{N / 2+1}\right) .
$$


Lemma 2.9. Keep the assumptions and notations of 2.9.

1) If $N$ is odd then $\rho\left(d_{a}\right)=g^{a} \otimes d_{a}$, for any $0 \leq a \leq N-1$.

2) If $N$ is even then $\rho\left(d_{a}\right)=g^{a} \otimes d_{a}$, for any $0 \leq a \leq N / 2$.

Moreover we have $\rho\left(d_{1} d_{N / 2}\right)=g^{1+N / 2} \otimes d_{1} d_{N / 2}+(x g-q g x) \otimes d_{1}$, where $x=\xi\left(d_{1} \otimes d_{N / 2-1}\right)$.

Proof. 1) and the first part of 2) follow by AMStu, Lemma 3.23]. Let $t=N / 2$. Then, for any $0 \leq i \leq 1,0 \leq j \leq t$ such that $0<i+j<1+t$, we have $1 \leq(1+t)-(i+j) \leq 1+t-1=$ $N / 2$. Since $(1-i)+(t-j)=(1+t)-(i+j)$, by (23) we have that $\xi\left(d_{1-i} \otimes d_{t-j}\right)=0$ unless $(1+t)-(i+j)=1+t-1=N / 2$ that is $i+j=1$, i.e. $(i, j)=(0,1)$ or $(i, j)=(1,0)$. Hence, by (19), for $a=1, b=t=N / 2$, we have

$$
\begin{aligned}
& \rho\left(d_{1} d_{N / 2}\right) \\
= & \sum g\left(d_{N / 2}\right)_{\langle-1\rangle} \otimes d_{1}\left(d_{N / 2}\right)_{\langle 0\rangle}+\xi\left(d_{1} \otimes d_{N / 2-1}\right) g \otimes d_{1}-q g \xi\left(d_{1} \otimes d_{N / 2-1}\right) \otimes d_{1} \\
= & g^{1+N / 2} \otimes d_{1} d_{N / 2}+\xi\left(d_{1} \otimes d_{N / 2-1}\right) g \otimes d_{1}-q g \xi\left(d_{1} \otimes d_{N / 2-1}\right) \otimes d_{1} .
\end{aligned}
$$

Lemma 2.10. Keep the assumptions and notations of 2.9. If either $N$ is odd or $N$ is even and $x=0$ (e.g. $N / 2$ is even or $H$ is cosemisimple) then

$$
\rho\left(d_{a}\right)=g^{a} \otimes d_{a}
$$

for any $0 \leq a \leq N-1$. Moreover, in this case, $R=R_{q}(H, g, \chi)$ is a quantum line.

Proof. Recall that by Lemma 2.9, we have that

- If $N$ is odd then $\rho\left(d_{a}\right)=g^{a} \otimes d_{a}$, for any $0 \leq a \leq N-1$.

- If $N$ is even then $\rho\left(d_{a}\right)=g^{a} \otimes d_{a}$, for any $0 \leq a \leq N / 2$.

Thus assume that $N$ is even and $x=0$.

If $N / 2+1 \leq a+b \leq N$, and $0<i+j<a+b$, since $(a-i)+(b-j)=(a+b)-(i+j)$, we have $0<(a-i)+(b-j)<N$ so that $\xi\left(d_{a-i} \otimes d_{b-j}\right)=0$ unless $(a-i)+(b-j)=N / 2$ in view of (23). We have

$$
\xi\left(d_{a-i} \otimes d_{b-j}\right)=\frac{1}{(a-i)_{q} !(b-j)_{q} !} \xi\left(y^{a-i} \otimes y^{b-j}\right) \stackrel{29}{=}=(N / 2-1)_{q} ! x=0 .
$$

Summing up, we have obtained that

$$
\xi\left(d_{a-i} \otimes d_{b-j}\right)=0, \text { if } N / 2+1 \leq a+b \leq N, 0<i+j<a+b .
$$

Therefore, by (19), we have

$$
\rho\left(d_{a} d_{b}\right)=\sum\left(d_{a}\right)_{\langle-1\rangle}\left(d_{b}\right)_{\langle-1\rangle} \otimes\left(d_{a}\right)_{\langle 0\rangle}\left(d_{b}\right)_{\langle 0\rangle} \text { for any } N / 2+1 \leq a+b \leq N .
$$

Since $\rho\left(d_{a}\right)=g^{a} \otimes d_{a}$, for any $0 \leq a \leq N / 2$, and since $d_{a} d_{b}=\left(\begin{array}{c}a+b \\ a\end{array}\right)_{q} d_{a+b}$, it is clear that the displayed relation above holds also for any $0 \leq a+b \leq N / 2$. Thus, by applying AMStu, Lemma 3.23], we obtain that $\rho\left(d_{a}\right)=g^{a} \otimes d_{a}$, for any $0 \leq a \leq N-1$. From this we infer that $\rho\left(y^{a}\right)=g^{a} \otimes y^{a}$, for any $0 \leq a \leq N-1$.

This fact entails that the multiplication $m$ of $R$ is left $H$-colinear and hence, by Theorem 2.4. $R=R_{q}(H, g, \chi)$ is a quantum line.

Proposition 2.11. Keep the assumptions and notations of 2.9 and assume that $H$ is either f.d. or cosemisimple.

1) Then, regardless to the parity of $N$, there exists $\lambda(N) \in K$ such that

$$
\xi\left(y \otimes y^{N-1}\right)=\xi\left(y^{2} \otimes y^{N-2}\right)=\cdots=\xi\left(y^{N-1} \otimes y\right)=\lambda(N)\left(1_{H}-g^{N}\right) .
$$

Moreover $(H, g, \chi, \lambda(N))$ is a compatible datum for $q$ and $Y^{N}=\lambda(N)\left(1_{R \#_{\xi} H}-\Gamma^{N}\right)$. 
2) If either $N$ is odd or $N$ is even and $x=0$ (e.g. $N / 2$ is even or $H$ is cosemisimple), then, for any $a, b \in \mathbb{N}$, we have

$$
\xi\left(y^{a} \otimes y^{b}\right)= \begin{cases}1 & \text { for } a+b=0 \\ \lambda(N)\left(1_{H}-g^{N}\right) & \text { for } a+b=N, a \neq 0, b \neq 0 \\ 0 & \text { otherwise }\end{cases}
$$

and $R=R_{q}(H, g, \chi)$ is a quantum line.

Proof. 1) By Lemma 2.6, we have $x^{2}=0$. By [AMStu, Corollary 3.22], there exists $\lambda(N) \in K$ such that

$$
Y^{N}=\lambda(N)\left(1_{R \#_{\xi} H}-\Gamma^{N}\right)=1_{R} \otimes \lambda(N)\left(1_{H}-g^{N}\right)
$$

where $\lambda(N)=0$ whenever $g^{N}=1_{H}$. Thus, by (31), we get $\xi\left(y \otimes y^{N-1}\right)=\lambda(N)\left(1_{H}-g^{N}\right)$.

By [AMStu, Theorem 3.29], there exists $\lambda(N)^{\prime}$ such that $\left(H, g, \chi, \lambda(N)^{\prime}\right)$ is a compatible datum for $q$ and $Y^{N}=\lambda(N)^{\prime}\left(1_{R \#_{\xi} H}-\Gamma^{N}\right)$. Since $\lambda(N)=0$ whenever $g^{N}=1_{H}$, we get $\lambda(N)^{\prime}=\lambda(N)$.

2) First of all, we point out that, in view of Theorem 2.4, $y^{n}=0$ for any $n \geq N$, so that we can assume $0 \leq a, b \leq N-1$.

Let $0 \leq a, b \leq N-1$. Then, from (23), we infer that

$$
\xi\left(y^{a} \otimes y^{b}\right)=0 \text { unless } a+b=0, \frac{N}{2}, N, \frac{3 N}{2},
$$

whenever they make sense.

Now, if $N$ is odd, the first assertion holds true in view of 1).

Assume $N$ is even and $x=0$. The first assertion holds true in view of 1),(29) and (32).

The last statement follows in view of Lemma 2.10.

Lemma 2.12. AMStu, Lemma 3.8] Let $H$ be a Hopf algebra, let $A$ be a bialgebra and let $\sigma: H \rightarrow A$ be an injective morphism of bialgebras having a retraction $\pi: A \rightarrow H$ (i.e. $\pi \sigma=H$ ) that is an $H$-bilinear coalgebra map. Let $(R, m, u, \delta, \varepsilon)$ be the pre-bialgebra in ${ }_{H}^{H} \mathcal{Y D}$ associated to $(A, \pi, \sigma)$ with corresponding cocycle $\xi$. Then, for every $r \in R$ and $h \in H$, we have

$$
\pi(r \sigma(h))=\varepsilon(r) h .
$$

Moreover the following assertions are equivalent:

(1) $\xi=\varepsilon \otimes \varepsilon$.

(2) $\pi: A \rightarrow H$ is a bialgebra homomorphism. $R$.

(3) $R$ is a braided bialgebra in ${ }_{H}^{H} \mathcal{Y D}$ and $R \# \xi H=R \# H$ is the Radford-Majid bosonization of

Corollary 2.13. Keep the assumptions and notations of 2.2 and assume that $H$ is either f.d. or cosemisimple. Then there exists a $\lambda(N) \in K$ such that (33) holds and $(H, g, \chi, \lambda(N))$ is a compatible datum for $q$.

If either $N$ is odd or $N$ is even and $x=0$, then the following assertions are equivalent:

(a) $\xi=\varepsilon \otimes \varepsilon$.

(b) The compatible datum $(H, g, \chi, \lambda(N))$ is trivial.

(c) $R$ is a braided bialgebra (in fact a quantum line) in ${ }_{H}^{H} \mathcal{Y D}$ and $B=R \#_{\xi} H$ is the RadfordMajid bosonization of $R$.

Proof. By Lemma 2.8, the first assertion holds.

$(a) \Leftrightarrow(b)$ By Proposition 2.11, we have that $\xi=\varepsilon \otimes \varepsilon$ if and only if $\lambda(N)\left(1_{H}-g^{N}\right)=0$. This condition is equivalent to triviality of the compatible datum.

$(a) \Leftrightarrow(c)$ It follows by applying Lemma 2.12 to $(B, \pi, \sigma)$ where $\pi: B \rightarrow H, \pi(r \# h)=\varepsilon(r) h$ and $\sigma: H \rightarrow B, \sigma(h)=1_{R} \# h$ (see also Definition 1.11 and Remark 1.12).

Theorem 2.14. Let $H$ be a Hopf algebra over a field $K$. Let $A$ be a bialgebra and let $\sigma: H \rightarrow A$ be an injective morphism of bialgebras having a retraction $\pi: A \rightarrow H$ (i.e. $\pi \sigma=\operatorname{Id}_{H}$ ) that is an $H$-bilinear coalgebra map. Let $(R, m, u, \delta, \varepsilon)$ be the pre-bialgebra in ${ }_{H}^{H} \mathcal{Y D}$ associated to $(A, \pi, \sigma)$ and let $\xi$ be the corresponding cocycle. Then the morphism $\omega: R \#_{\xi} H \rightarrow A, \omega(r \otimes h)=r \sigma(h)$ is a bialgebra isomorphism. 
Assume that

- $H$ is either f.d. or cosemisimple;

- $R$ is an $N$-dimensional thin coalgebra where $P(R)=K y$.

Let $g \in H$ and $\chi \in H^{*}$ be such that $(H, g, \chi)$ is the Yetter-Drinfeld datum associated to $y$ and let $q=\chi(g)$.

Then there exists $\lambda(N) \in K$ such that $(H, g, \chi, \lambda(N))$ is a compatible datum for $q$ and $y \cdot{ }^{N}{ }^{N}=$ $\lambda(N)\left(1_{H}-\sigma(g)^{N}\right)$.

Moreover the following assertions are equivalent:

(a) $m$ is left $H$-colinear.

(b) $N$ is odd or $\xi\left(y \otimes y^{\cdot R} N / 2-1\right)=0$.

(c) The $n$-th iterated power $y^{\cdot{ }^{n}}{ }^{n}$ of $y$ in $R$ and the $n$-th iterated power $y^{\cdot{ }^{n}}$ of $y$ in $A$ coincides for every $0 \leq n \leq N-1$.

(d) $R=R_{q}(H, g, \chi)$ is a quantum line.

Furthermore, if these conditions are satisfied, for any $a, b \in \mathbb{N}$, we have

$$
\xi\left(y^{a} \otimes y^{b}\right)= \begin{cases}1 & \text { for } a+b=0 \\ \lambda(N)\left(1_{H}-g^{N}\right) & \text { for } a+b=N, a \neq 0, b \neq 0 \\ 0 & \text { otherwise }\end{cases}
$$

and the following assertions are equivalent:

(1) $\xi=\varepsilon \otimes \varepsilon$.

(2) The compatible datum $(H, g, \chi, \lambda(N))$ is trivial i.e. $\lambda(N)=0$.

(3) $A$ is the Radford-Majid bosonization of $R$.

(4) $\pi$ is a bialgebra homomorphism.

Proof. The first assertion follows by Definitions 1.11.

Assume that $H$ is either f.d. or cosemisimple and that $R$ is an $N$-dimensional thin coalgebra where $P(R)=K y$.

The second assertion follows by Lemma 2.8 which implies also that $\xi\left(y \otimes y^{N-1}\right)=\lambda(N)\left(1_{H}-g^{N}\right)$.

Note that the assumptions of 2.2 are fulfilled so that we can keep also the notations therein. In particular, we can consider a divided power sequence of non-zero elements in $R d_{0}=1_{R}, d_{1}=$ $y, \ldots, d_{N-1}$ which satisfies the conditions stated in 2.2. By (29) in Proposition 2.8, we have $\xi\left(y \otimes y^{N / 2-1}\right)=(N / 2-1)_{q} ! x$ so that $\xi\left(y \otimes y^{N / 2-1}\right)=0$ is equivalent to $x=0$.

$(d) \Rightarrow(a)$ is trivial.

$(a) \Rightarrow(b)$ Assume that $N$ is even.

If $N=2$, then, by Lemma 2.6, $x=0$.

Assume $N \neq 2$. Then, by Lemma 2.9, we have

$$
\rho\left(d_{N / 2}\right)=g^{N / 2} \otimes d_{N / 2}, \quad \text { and } \quad \rho\left(d_{1} d_{N / 2}\right)=g^{1+N / 2} \otimes d_{1} d_{N / 2}+(x g-q g x) \otimes d_{1} .
$$

By hypothesis $\rho\left(d_{1} d_{N / 2}\right)=g^{1+N / 2} \otimes d_{1} d_{N / 2}$, so that $(x g-q g x) \otimes d_{1}=0$ that is $x g=q g x$. By Lemma 2.6, we also have $x g+g x=0$ whence $g(q x)=q g x=x g=-g x$. By multiplying on the left by $g^{-1}$, we get $q x=-x$ which implies $x=0$ since $q \neq-1$ (otherwise $N=o(q)=2$ ).

$(b) \Rightarrow(c)$ By Proposition 2.8, (b) implies $N$ is odd or $\xi\left(y \otimes y^{N / 2-1}\right)=0$. By AMStu, Theorem 3.30], (c) holds true.

$(c) \Rightarrow(b)$ Assume that $N$ is even. By Proposition 2.7 we have that $Y^{N / 2}=X+y^{N / 2} \otimes 1_{H}$. Thus

$$
y^{\cdot{ }_{A} N / 2}=\omega(Y)^{\cdot{ }_{A} N / 2}=\omega\left(Y^{N / 2}\right)=\omega(X)+\omega\left(y^{\cdot{ }_{R} N / 2} \otimes 1_{H}\right)=\omega(X)+y^{\cdot{ }_{R} N / 2}
$$

so that $\omega(X)=0$ and hence $X=0$. Since $X=(N / 2-1)_{q} ! \sigma(x)$ and $o(q)=N$ we obtain $\sigma(x)=0$ which entails $x=0$.

$(b) \Rightarrow(d)$ follows by Proposition 2.11 which also implies that $\xi$ fulfills (34).

$(1) \Leftrightarrow(2)$ follows by Corollary 2.13.

$(1) \Leftrightarrow(3) \Leftrightarrow(4)$ follows by Lemma 2.12 . 
Note that in the following result $H$ is a cosemisimple Hopf algebra and we require the existence of a retraction $\pi$ which is an. $H$-bilinear coalgebra map. In Theorem $3.8 H$ is assumed to be both cosemisimple and finite dimensional. In this case such a retraction always exists.

Corollary 2.15. Let $H$ be a cosemisimple Hopf algebra over a field $K$. Let $A$ be a bialgebra and let $\sigma: H \rightarrow A$ be an injective morphism of bialgebras having a retraction $\pi: A \rightarrow H$ (i.e. $\left.\pi \sigma=\operatorname{Id}_{H}\right)$ that is an $H$-bilinear coalgebra map. Let $(R, m, u, \delta, \varepsilon)$ be the pre-bialgebra in ${ }_{H}^{H} \mathcal{Y D}$ associated to $(A, \pi, \sigma)$ and let $\xi$ be the corresponding cocycle. Then the morphism $\omega: R \# \xi H \rightarrow A$, $\omega(r \otimes h)=r \sigma(h)$ is a bialgebra isomorphism.

Assume that $R$ is an $N$-dimensional thin coalgebra where $P(R)=K y$ (hence $\sigma(H)=A_{0}$ ).

Let $g \in H$ and $\chi \in H^{*}$ be such that $(H, g, \chi)$ is the Yetter-Drinfeld datum associated to $y$ and let $q=\chi(g)$.

Then we have that:

a) There exists $\lambda(N) \in K$ such that $(H, g, \chi, \lambda(N))$ is a compatible datum for $q$ and $y^{\cdot}{ }^{N}=$ $\lambda(N)\left(1_{H}-\sigma(g)^{N}\right)$.

b) The $n$-th iterated power $y^{\cdot{ }^{n}}$ of $y$ in $R$ and the $n$-th iterated power $y^{\cdot{ }^{n}}$ of $y$ in $A$ coincides for every $0 \leq n \leq N-1$.

c) $R=R_{q}(H, g, \chi)$ is a quantum line.

Moreover, for any $a, b \in \mathbb{N}$, we have

$$
\xi\left(y^{a} \otimes y^{b}\right)= \begin{cases}1 & \text { for } a+b=0 \\ \lambda(N)\left(1_{H}-g^{N}\right) & \text { for } a+b=N, a \neq 0, b \neq 0 \\ 0 & \text { otherwise }\end{cases}
$$

and the following assertions are equivalent:

(1) $\xi=\varepsilon \otimes \varepsilon$.

(2) The compatible datum $(H, g, \chi, \lambda(N))$ is trivial.

(3) $A$ is the Radford-Majid bosonization of $R$.

(4) $\pi$ is a bialgebra homomorphism.

Proof. It follows by 7) in Lemma 2.6 and by Theorem 2.14.

Note that, in view of Lemma 1.16, we have $\sigma(H)=A_{0}$.

\section{Ad-InVARIANT INTEGRALS}

3.1. Recall from [AMSte, Definition 2.7] that an $a d$-invariant integral for a Hopf algebra $H$ is a $K$-linear map $\gamma: H \rightarrow K$ such that

$$
\sum h_{(1)} \gamma\left(h_{(2)}\right)=1_{H} \gamma(h), \quad \gamma\left(1_{H}\right)=1_{K}, \quad \sum \gamma\left[h_{(1)} x S_{H}\left(h_{(2)}\right)\right]=\varepsilon_{H}(h) \gamma(x),
$$

for any $h, x \in H$. From AMSte, Theorem 2.27], any semisimple and cosemisimple Hopf algebra (e.g. f.d. cosemisimple and $\operatorname{char}(K)=0$ ) has such an integral.

REMARK 3.2. The group algebra, which is in general not semisimple, always admits an ad-invariant integral.

Lemma 3.3. Let $\chi \in H^{*}$ be a character of a Hopf algebra $H$. Let $n \in \mathbb{N}$ and let $z \in H$. Then

$$
\chi^{n}(h) z=\sum h_{(1)} z S\left(h_{(2)}\right), \text { for every } h \in H \Longleftrightarrow h z=z \varphi_{H}^{n}(h), \text { for every } h \in H
$$

where $\varphi_{H}$ is the automorphism of Lemma 1.14 .

Proof. Assume $\chi^{n}(h) z=\sum h_{(1)} z S\left(h_{(2)}\right)$. Then

$$
h z=\sum h_{(1)} z S\left(h_{(2)}\right) h_{(3)}=\sum \chi^{n}\left(h_{(1)}\right) z h_{(2)}=z \sum \chi^{n}\left(h_{(1)}\right) h_{(2)}=z \varphi_{H}^{n}(h) .
$$

The converse also holds true, in fact

$$
\sum h_{(1)} z S\left(h_{(2)}\right)=z \varphi_{H}^{n}\left(h_{(1)}\right) S\left(h_{(2)}\right)=z \sum \chi^{n}\left(h_{(1)}\right) h_{(2)} S\left(h_{(3)}\right)=z \chi^{n}(h) .
$$


LEMmA 3.4. Let $(H, g, \chi)$ be a Yetter-Drinfeld datum and assume that $H$ has an ad-invariant integral $\gamma$ (e.g. $H$ f.d. cosemisimple). If $g^{N} \neq 1_{H}$, then the following assertions are equivalent:

(a) $\chi^{N}(h)\left(1_{H}-g^{N}\right)=\sum h_{(1)}\left(1_{H}-g^{N}\right) S\left(h_{(2)}\right)$, for every $h \in H$.

(b) $\chi^{N}=\varepsilon_{H}$ and $g^{N} \in Z(H)$.

Proof. Assume that $g^{N} \neq 1$.

$(a) \Rightarrow(b)$ By applying $\gamma$ to both sides of equality in $(a)$, we get

$$
\begin{aligned}
\gamma\left[\chi^{N}(h)\left(1_{H}-g^{N}\right)\right] & =\gamma\left[\sum h_{(1)}\left(1_{H}-g^{N}\right) S\left(h_{(2)}\right)\right] \text { i.e. } \\
\chi^{N}(h) \lambda\left(1_{H}-g^{N}\right) & =\varepsilon_{H}(h) \lambda\left(1_{H}-g^{N}\right) .
\end{aligned}
$$

Since $g^{N} \in G(H)$ and $g^{N} \neq 1_{H}$, we infer $\gamma\left(g^{N}\right)=0$ so that we get $\chi^{N}(h)=\varepsilon_{H}(h)$ for any $h \in H$ and hence $\chi^{N}=\varepsilon_{H}$. By equality in $(a)$ and by Lemma 3.3 applied to the case $z=1_{H}-g^{N}$, we deduce $h\left(1_{H}-g^{N}\right)=\left(1_{H}-g^{N}\right) \varphi_{H}^{N}(h)$, for every $h \in H$.

Since $\varphi_{H}^{N}(h)=\sum \chi^{N}\left(h_{(1)}\right) h_{(2)}=h$, we get that $g^{N} \in Z(H)$.

$(b) \Rightarrow(a)$ It is trivial.

Proposition 3.5. Let $H$ be a Hopf algebra endowed with an ad-invariant integral (e.g. $H$ f.d. cosemisimple). Then a compatible datum for $q$ is exactly a quadruple $(H, g, \chi, \lambda(N))$, where

- $(H, g, \chi)$ is a Yetter-Drinfeld datum for $q$,

- $\lambda(N) \in K$ is arbitrary if

$$
\chi^{N}=\varepsilon_{H}, \quad \text { and } \quad g^{N} \in Z(H) \backslash\left\{1_{H}\right\},
$$

while $\lambda(N)=0$ otherwise.

Proof. By definition a compatible datum for $q$ is a quadruple $(H, g, \chi, \lambda(N))$ such that $(H, g, \chi)$ is a Yetter-Drinfeld datum for $q, \lambda(N) \in K$ and $\lambda(N)=0$ if

$$
g^{N}=1_{H}, \quad \text { or } \quad \chi^{N}(h)\left(1_{H}-g^{N}\right) \neq \sum h_{(1)}\left(1_{H}-g^{N}\right) S h_{(2)}, \text { for some } h \in H,
$$

while $\lambda(N)$ is an arbitrary otherwise. Equivalently $\lambda(N) \in K$ is arbitrary if

$$
g^{N} \neq 1_{H}, \quad \text { and } \quad \chi^{N}(h)\left(1_{H}-g^{N}\right)=\sum h_{(1)}\left(1_{H}-g^{N}\right) S h_{(2)}, \text { for every } h \in H,
$$

while $\lambda(N)=0$ otherwise.

The conclusion follows by observing that, by Lemma 3.4, the displayed conditions are equivalent to $\chi^{N}=\varepsilon_{H}$ and $g^{N} \in Z(H) \backslash\left\{1_{H}\right\}$.

REMARK 3.6. In the case when $H=K G(H)$ and $G(H)$ is a finite abelian group, in view of Proposition 3.5, our definition of a compatible datum agrees with [AS, page 679].

Proposition 3.7. Keep the assumptions and notations of 2.2. If $H$ has an ad-invariant integral (e.g. H f.d. cosemisimple), then, there exists $\lambda(N) \in K$ such that, for any $a, b \in \mathbb{N}$, we have

$$
\xi\left(y^{a} \otimes y^{b}\right)= \begin{cases}1 & \text { for } a+b=0 \\ \lambda(N)\left(1_{H}-g^{N}\right) & \text { for } a+b=N, a \neq 0, b \neq 0 \\ 0 & \text { otherwise. }\end{cases}
$$

and $(H, g, \chi, \lambda(N))$ is a compatible datum for $q$. Moreover, in this case, $R=R_{q}(H, g, \chi)$ is a quantum line and, if $\xi \neq \varepsilon \otimes \varepsilon$, we have

$$
\chi^{N}=\varepsilon_{H} \quad \text { and } \quad g^{N} \in Z(H) \backslash\left\{1_{H}\right\} .
$$

Proof. Assume that $H$ has an ad-invariant integral. In particular this is a total integral and hence $H$ is cosemisimple so that, by Proposition 2.11, we get the first statement. Assume $\xi \neq \varepsilon \otimes \varepsilon$. By Corollary 2.13, this means $\lambda(N)\left(1_{H}-g^{N}\right) \neq 0$ so that $\lambda(N) \neq 0$ and $g^{N} \neq 1_{H}$. By Proposition 3.5, we conclude. 
TheOREM 3.8. Let $A$ be a bialgebra over a field $K$. Suppose that the coradical $H$ of $A$ is a f.d. subbialgebra of $A$ with antipode. Then $A$ is a Hopf algebra and there is a retraction $\pi: A \rightarrow H$ (i.e. $\pi \sigma=H$ where $\sigma: H \rightarrow A$ is the canonical injection) that is an $H$-bilinear coalgebra map. Let $(R, m, u, \delta, \varepsilon)$ be the pre-bialgebra in ${ }_{H}^{H} \mathcal{Y D}$ associated to $(A, \pi, \sigma)$ with corresponding cocycle $\xi$.

Assume that $R$ is an $N$-dimensional thin coalgebra where $P(R)=K y$.

Then there exist

- a primitive $N$-th root of unit $q$,

- $g \in H, \chi \in H^{*}, \lambda(N) \in K$ so that $(H, g, \chi, \lambda(N))$ is a compatible datum for $q$

such that

1) $R=R_{q}(H, g, \chi)$ is a quantum line spanned by $y$.

2) The $n$-th iterated power of $y$ in $R$ and the $n$-th iterated power of $y$ in $A$ coincide for every $0 \leq n \leq N-1$.

3)

$$
\xi\left(y^{a} \otimes y^{b}\right)= \begin{cases}1 & \text { for } a+b=0 \\ \lambda(N)\left(1_{H}-g^{N}\right) & \text { for } a+b=N, a \neq 0, b \neq 0 \\ 0 & \text { otherwise. }\end{cases}
$$

Moreover $A$ is a Hopf algebra with basis

$$
\left\{y^{i} \sigma(h) \mid 0 \leq i \leq N-1, h \in B(H)\right\},
$$

algebra structure given by

$$
\begin{aligned}
y^{N} & =\lambda(N)\left(1_{A}-\Gamma^{N}\right), \\
\sigma(h) y^{a} & =y^{a} \sigma\left[\varphi_{H}^{a}(h)\right] \text { for any } a \in \mathbb{N}, \text { and } h \in H
\end{aligned}
$$

and coalgebra structure given by

$$
\Delta_{A}(y)=y \otimes 1_{A}+\Gamma \otimes y .
$$

Here $\varphi_{H}: H \rightarrow H$ denotes the algebra automorphism of $H$ defined by $\varphi_{H}(h)=\sum \chi\left(h_{(1)}\right) h_{(2)}$ and $\Gamma=\sigma(g)$.

Furthermore, if $y^{N}=\lambda(N)\left(1_{A}-\Gamma^{N}\right) \neq 0$, then

$$
\chi^{N}=\varepsilon_{H} \quad \text { and } \quad g^{N} \in Z(H) \backslash\left\{1_{H}\right\} .
$$

Finally, if $Z(H)=\left\{1_{H}\right\}$, then $\pi$ is also an algebra homomorphism and hence $A$ is the RadfordMajid bosonization of $R$.

Proof. In view of [AMStu, Theorem 4.5] it remains to prove the last two sentences.

The second last one follows by Proposition 3.7 and Theorem 2.14 .

Assume that $Z(H)=\left\{1_{H}\right\}$. Then, by the foregoing, we get $\lambda(N)\left(1_{H}-g^{N}\right)=0$ so that the compatible datum $(H, g, \chi, \lambda(N))$ is trivial. The conclusion follows in view of Theorem 2.14.

\section{Ore Extensions}

Recall that for a $K$-algebra $A$, an algebra endomorphism $\varphi: A \rightarrow A$ and a linear map $\delta: A \rightarrow A$ such that $\delta(a b)=a \delta(b)+\delta(a) \varphi(b)$, for every $a, b \in A$ (namely $\delta$ is a $\varphi$-derivation), the Ore extension (or Skew Polinomial Ring) $A[X, \varphi, \delta]$ is $A[X]$ as an abelian group, with multiplication induced by

$$
a X=\delta(a)+X \varphi(a), \text { for every } a \in A .
$$

The Ore extension $A[X, \varphi, \delta]$ fulfills the following universal property.

Lemma 4.1. Let $A[X, \varphi, \delta]$ be an Ore extension of $A$ and let $i: A \rightarrow A[X, \varphi, \delta]$ be the canonical inclusion. Let $B$ be an algebra, let $f: A \rightarrow B$ be an algebra homomorphism and let $b \in B$ be such that

$$
f(a) b=f[\delta(a)]+b f[\varphi(a)], \text { for every } a \in A .
$$

Then, there exists a unique algebra homomorphism $\bar{f}: A[X, \varphi, \delta] \rightarrow B$ such that

$$
\bar{f}(X)=b \quad \text { and } \quad \bar{f} \circ i=f .
$$


THEOREM 4.2. Let $q$ be a primitive $N$-th root of unity.

For any compatible datum $(H, g, \chi, \lambda(N))$ for $q$, there exist a Hopf algebra

$$
\mathcal{O}=\mathcal{O}(H, g, \chi, \lambda(N))
$$

an injective Hopf algebra map $\sigma_{\mathcal{O}}: H \rightarrow \mathcal{O}$ and an element $y \in \mathcal{O}$ such that

$$
\left\{y^{i} \sigma_{\mathcal{O}}(h) \mid 0 \leq i \leq N-1, h \in \mathcal{B}(H)\right\},
$$

is a basis for $\mathcal{O}$, where $\mathcal{B}(H)$ is a basis of $H$; moreover the algebra structure of $\mathcal{O}$ is given by

$$
\begin{aligned}
y^{N} & =\lambda(N)\left(1_{\mathcal{O}}-\Gamma^{N}\right), \text { where } \Gamma=\sigma_{\mathcal{O}}(g) \\
\sigma_{\mathcal{O}}(h) y^{a} & =y^{a} \sigma_{\mathcal{O}}\left[\varphi_{H}^{a}(h)\right] \text { for any } a \in \mathbb{N}, \text { and } h \in H,
\end{aligned}
$$

and the coalgebra structure is given by

$$
\Delta_{\mathcal{O}}(y)=y \otimes 1_{\mathcal{O}}+\Gamma \otimes y .
$$

Here $\varphi_{H}: H \rightarrow H$ denotes the algebra automorphism of $H$ defined by $\varphi_{H}(h)=\sum \chi\left(h_{(1)}\right) h_{(2)}$.

i) Let

$$
p: \mathcal{O} \rightarrow H, p\left[y^{n} \sigma_{\mathcal{O}}(h)\right]=\delta_{n, 0} h, \text { for every } 0 \leq n \leq N-1, h \in \mathcal{B}(H) .
$$

Then $p$ is an $H$-bilinear coalgebra (not necessarily algebra) retraction $\left(p \sigma_{\mathcal{O}}=H\right.$ ) of $\sigma$. Moreover the pre-bialgebra in ${ }_{H}^{H} \mathcal{Y} \mathcal{D}$ associated to $\left(\mathcal{O}, p, \sigma_{\mathcal{O}}\right)$ is $(R, m, u, \delta, \varepsilon)$ with corresponding cocycle $\xi$ where

1) $R=R_{q}(H, g, \chi)$ is a braided bialgebra in ${ }_{H}^{H} \mathcal{Y} \mathcal{D}$, in fact a quantum line spanned by $y$ of dimension $N$ and the $N$-th power of $y$ in $R$ is zero.

2) for any $0 \leq n \leq N-1$, the $n$-th power of $y$ in $R$ coincides with the $n$-th power of $y$ in $\mathcal{O}$, namely $y^{n}$.

3) for any $0 \leq a, b \leq N-1$, we have

$$
\xi\left(y^{a} \otimes y^{b}\right)= \begin{cases}1 & \text { for } a+b=0 \\ \lambda(N)\left(1_{H}-g^{N}\right) & \text { for } a+b=N \\ 0 & \text { otherwise. }\end{cases}
$$

Furthermore the map

$$
\omega: R_{q}(H, g, \chi) \# \xi H \rightarrow \mathcal{O}(H, g, \chi, \lambda(N)), \omega(r \otimes h)=r \sigma_{\mathcal{O}}(h)
$$

is a Hopf algebra isomorphism.

ii) Let $B$ be a bialgebra, let $f: H \rightarrow B$ be a bialgebra homomorphism and $b \in B$ be such that

$$
\begin{gathered}
f(h) b=b f\left[\varphi_{H}(h)\right], \text { for every } h \in H, \\
b^{N}=\lambda(N)\left(1-f(g)^{N}\right), \quad \Delta_{B}(b)=b \otimes 1_{B}+f(g) \otimes b .
\end{gathered}
$$

Then there exists a unique bialgebra homomorphism $\widehat{f}: \mathcal{O} \rightarrow B$ such that $\widehat{f} \circ \sigma_{\mathcal{O}}=f$ and $\widehat{f}(y)=b$.

Proof. Let $w=\lambda(N)\left(1-g^{N}\right)$. We will endow the Ore extension $O=H\left[X, \varphi_{H}, 0\right]$ with a bialgebra structure and we will quotient it by a suitable ideal. By applying the universal property of Ore extensions to the case $B=O \otimes O, f=(i \otimes i) \Delta_{H}$ and $b=X \otimes 1_{O}+g \otimes X$ one can prove that there exists a unique algebra homomorphism $\Delta_{O}: O \rightarrow O \otimes O$ such that

$$
\Delta_{O}(X)=b=X \otimes 1_{O}+g \otimes X \quad \text { and } \quad \Delta_{O} \circ i=(i \otimes i) \Delta_{H} .
$$

Let us apply once more the universal property of Ore extensions to obtain a counit. We do it in the case $B=K, f=\varepsilon_{H}$ and $b=0$. Therefore there exists a unique algebra homomorphism $\varepsilon_{O}: O \rightarrow K$ such that

$$
\varepsilon_{O}(X)=b=0 \quad \text { and } \quad \varepsilon_{O} \circ i=\varepsilon_{H} .
$$

Obviously $\left(O, \Delta_{O}, \varepsilon_{O}\right)$ is a coalgebra such that $O$ becomes a bialgebra. Let us prove that $O$ has an antipode. By the universal property of $O$, there exists a unique algebra homomorphism $S_{O}: O \rightarrow O^{o p}$ such that

$$
S_{O}(X)=b \quad \text { and } \quad S_{O} \circ i=f
$$


where $f: H \rightarrow O^{o p}$ is the algebra homomorphism defined by $f=i S_{H}$ and $b=-g^{-1} X \in O$. In conclusion $S_{O}$ is an antipode for $O$ which becomes a Hopf algebra. From $\Delta_{O} \circ i=(i \otimes i) \Delta_{H}$ and $\varepsilon_{O} \circ i=\varepsilon_{H}$, we get that $i$ is a bialgebra and hence a Hopf algebra map. The two-sided ideal $J:=\left(X^{N}-w\right)$ of $O$ is a Hopf ideal. Therefore we are led to consider the Hopf algebra

$$
\mathcal{O}=\mathcal{O}(H, g, \chi, \lambda(N))=\frac{H\left[X, \varphi_{H}, 0\right]}{\left(X^{N}-w\right)} .
$$

Let $\sigma_{\mathcal{O}}: H \rightarrow \mathcal{O}$ be the canonical map and set $y=X+J$. It is straightforward to prove that $\mathcal{O}$ fulfills the required properties.

i) It follows by [AMStu, Theorem 4.1] and Definitions 1.11.

ii) By the universal property of Ore extensions, if $B$ is an algebra, $f: H \rightarrow B$ is an algebra homomorphism and $b \in B$ is such that $f(h) b=b f\left[\varphi_{H}(h)\right]$, for every $h \in H$, then, there exists a unique algebra homomorphism $\bar{f}: H\left[X, \varphi_{H}, 0\right] \rightarrow B$ such that

$$
\bar{f}(X)=b \quad \text { and } \quad \bar{f} \circ i=f .
$$

Since

$$
\bar{f}\left(X^{N}-w\right)=b^{N}-\bar{f}\left[\lambda(N)\left(1-g^{N}\right)\right]=b^{N}-\lambda(N)\left(1-f(g)^{N}\right)=0
$$

we obtain an algebra homomorphism $\widehat{f}: \mathcal{O} \rightarrow B$. Clearly $\widehat{f} \circ \sigma_{\mathcal{O}}=f$ and $\widehat{f}(y)=b$.

Assume that $B$ is a bialgebra. From

$$
\begin{gathered}
(\bar{f} \otimes \bar{f}) \Delta_{O}(X)=(\bar{f} \otimes \bar{f})\left(X \otimes 1_{O}+g \otimes X\right)=b \otimes 1_{B}+f(g) \otimes b=\Delta_{B}(b)=\Delta_{B} \bar{f}(X) \\
(\bar{f} \otimes \bar{f}) \Delta_{O} i=(\bar{f} \otimes \bar{f})(i \otimes i) \Delta_{H}=(f \otimes f) \Delta_{H}=\Delta_{B} f=\Delta_{B} \bar{f} \circ i
\end{gathered}
$$

we get $\Delta_{B} \circ \bar{f}=(\bar{f} \otimes \bar{f}) \circ \Delta_{O}$. From $b \otimes 1_{B}+f(g) \otimes b=\Delta_{B}(b)$ we get $\varepsilon_{B}(b)=0$ and hence

$$
\varepsilon_{B} \bar{f}(X)=\varepsilon_{B}(b)=0=\varepsilon_{O}(X) \quad \text { and } \quad \varepsilon_{B} \bar{f} i=\varepsilon_{B} f=\varepsilon_{O} .
$$

Thus $\varepsilon_{B} \circ \bar{f}=\varepsilon_{O}$ so that $\bar{f}$ is a bialgebra homomorphism and hence $\widehat{f}$ is a bialgebra homomorphism.

Remark 4.3. Let $(H, g, \chi, \lambda(N))$ be a compatible datum for $q$ where $H$ is a cosemisimple Hopf algebra. Then, in view of Theorem 4.2 and of Lemma 1.16, $H$ is the coradical of $\mathcal{O}(H, g, \chi, \lambda(N))$.

Theorem 4.4. Let $H$ be a Hopf algebra over a field $K$. Let $A$ be a bialgebra and let $\sigma: H \rightarrow A$ be an injective morphism of bialgebras having a retraction $\pi: A \rightarrow H$ (i.e. $\pi \sigma=H$ ) that is an $H$-bilinear coalgebra map. Assume that either $H$ is f.d. or cosemisimple and that the coalgebra in the pre-bialgebra in ${ }_{H}^{H} \mathcal{Y D}$ associated to $(A, \pi, \sigma)$ is $N$-dimensional and thin.

Then there exists

- a primitive $N$-th root of unit $q$,

- $g \in H, \chi \in H^{*}, \lambda(N) \in K$

such that $(H, g, \chi, \lambda(N))$ is a compatible datum for $q$ and there is a bialgebra isomorphism $\widehat{\sigma}: \mathcal{O}(H, g, \chi, \lambda(N)) \rightarrow A$ such that $\widehat{\sigma} \circ \sigma_{\mathcal{O}}=\sigma$.

Proof. By [AMStu, Theorem 4.2], there exists $z \in A$ such that $A$ is a Hopf algebra with basis

$$
\left\{z^{i} \sigma(h) \mid 0 \leq i \leq N-1, h \in B(H)\right\},
$$

algebra structure given by $z^{N}=\lambda(N)\left(1_{A}-\Gamma^{N}\right), \sigma(h) z^{a}=z^{a} \sigma\left[\varphi_{H}^{a}(h)\right]$ for any $a \in \mathbb{N}$, and $h \in H$ and coalgebra structure given by $\Delta_{A}(z)=z \otimes 1_{A}+\Gamma \otimes z$. Here $\varphi_{H}: H \rightarrow H$ denotes the algebra automorphism of $H$ defined by $\varphi_{H}(h)=\sum \chi\left(h_{(1)}\right) h_{(2)}$ and $\Gamma=\sigma(g)$. By Theorem 4.2 applied to that case $(B, f, b):=(A, \sigma, z)$, there exists a unique bialgebra homomorphism

$$
\widehat{\sigma}: \mathcal{O}=\mathcal{O}(H, g, \chi, \lambda(N)) \rightarrow A
$$

such that $\widehat{\sigma} \circ \sigma_{\mathcal{O}}=\sigma$ and $\widehat{\sigma}(y)=z$. Clearly $\widehat{\sigma}$ is bijective as it sends bijectively a basis of $\mathcal{O}$ to a basis of $A$. 
TheOREM 4.5. Let A be a finite dimensional bialgebra over a field K. Suppose that the coradical $H$ of $A$ is a subbialgebra of $A$ with antipode. Then $A$ is a Hopf algebra and there is a retraction $\pi: A \rightarrow H$ (i.e. $\pi \sigma=H$ where $\sigma: H \rightarrow A$ denotes the canonical injection) that is an $H$ bilinear coalgebra map. Let $(R, m, u, \delta, \varepsilon)$ be the pre-bialgebra in ${ }_{H}^{H} \mathcal{Y} \mathcal{D}$ associated to $(A, \pi, \sigma)$ with corresponding cocycle $\xi$. Then the following assertions are equivalent:

(a) $\operatorname{dim} A_{1}=2 \operatorname{dim} H$.

(b) $R$ is thin.

Moreover, if one of these conditions is fulfilled, then there exists a primitive $N$-th root of unit $q, g \in H, \chi \in H^{*}, \lambda(N) \in K$ such that $(H, g, \chi, \lambda(N))$ is a compatible datum for $q$ and there is a bialgebra isomorphism $\widehat{\sigma}: \mathcal{O}(H, g, \chi, \lambda(N)) \rightarrow A$ such that $\widehat{\sigma} \circ \sigma_{\mathcal{O}}=\sigma$.

Proof. The first part follows by Theorem 3.8.

By Definitions 1.11, the morphism $\omega: R \#_{\xi} H \rightarrow A, \omega(r \otimes h)=r h$ is a bialgebra isomorphism. By [AMSte, Theorem 3.71], for every $n \in \mathbb{N}$, we have that $\left(R \#_{\xi} H\right)_{n}=R_{n} \otimes H$, where $\left(R_{n}\right)_{n \in \mathbb{N}}$ is the coradical filtration of $R$. Through $\omega$ we get $A_{n}=R_{n} H$ so that

$$
\operatorname{dim} H=\operatorname{dim} A_{0}=\operatorname{dim} R_{0} \cdot \operatorname{dim} H, \quad \operatorname{dim} A_{1}=\operatorname{dim} R_{1} \cdot \operatorname{dim} H .
$$

From the first chain of equalities we get that $\operatorname{dim} R_{0}=1$ so that $R$ is connected. Therefore $R$ is thin if and only if $\operatorname{dim} R_{1}=2$ i.e. $\operatorname{dim} A_{1}=2 \operatorname{dim} H$.

The last part of the statement follows by Theorem 4.4.

REMARK 4.6. Part of the foregoing theorem is contained in [AS, Corollary, page 673] where it is proved that if $A$ fulfills $(a)$ then it is generated as an algebra by $A_{1}$.

Lemma 4.7. Let $q$ be a primitive $N$-th root of unity.

Let $(H, g, \chi, \lambda(N))$ be a compatible datum for $q$ where $H$ is a cosemisimple Hopf algebra. We use the notations of Theorem 4.9. Let $z \in \mathcal{O}=\mathcal{O}(H, g, \chi, \lambda(N))$, be such that

$$
\Delta_{\mathcal{O}}(z)=z \otimes 1_{\mathcal{O}}+\gamma \otimes z, \gamma \in \sigma_{\mathcal{O}}(H) .
$$

Then $\gamma=\Gamma$ and $z:=y \alpha+\beta(1-\Gamma)$ for some $\alpha, \beta \in K$.

Proof. Recall that the map

$$
\omega: R_{q}(H, g, \chi) \#_{\xi} H \rightarrow \mathcal{O}(H, g, \chi, \lambda(N)), \omega(r \otimes h)=r \sigma_{\mathcal{O}}(h)
$$

is a Hopf algebra isomorphism. Since $R=R_{q}(H, g, \chi)$ is connected, in view of Lemma 1.16, we have $\mathcal{O}_{0}=H$ through $\omega$ where we identified $\sigma_{\mathcal{O}}(H)$ with $H$. Now $z \in \mathcal{O}_{0} \wedge_{\mathcal{O}} \mathcal{O}_{0}=\mathcal{O}_{1}$. By [AMSte, Theorem 3.71], we have that $\left(R \#_{\xi} H\right)_{1}=R_{1} \otimes H$. Through $\omega$, we get $\mathcal{O}_{1}=R_{1} H=P(R) H+H$. Hence

$$
z=y \alpha+\beta, \text { for some } \alpha, \beta \in H .
$$

From $\Delta_{\mathcal{O}}(z)=z \otimes 1_{\mathcal{O}}+\gamma \otimes z, \gamma \in G(H)$, we get

$$
\Delta_{\mathcal{O}}(y \alpha+\beta)=(y \alpha+\beta) \otimes 1_{\mathcal{O}}+\gamma \otimes(y \alpha+\beta) .
$$

Since $\Delta_{\mathcal{O}}(y)=y \otimes 1_{\mathcal{O}}+\Gamma \otimes y, \Gamma \in G(H)$, we obtain

$$
\begin{gathered}
\sum y \alpha_{(1)} \otimes \alpha_{(2)}+\sum \Gamma \alpha_{(1)} \otimes y \alpha_{(2)}+\Delta_{\mathcal{O}}(\beta)=y \alpha \otimes 1_{\mathcal{O}}+\beta \otimes 1_{\mathcal{O}}+\gamma \otimes y \alpha+\gamma \otimes \beta \text { i.e. } \\
\left\{\begin{array}{l}
\sum y \alpha_{(1)} \otimes \alpha_{(2)}=y \alpha \otimes 1_{\mathcal{O}} \\
\sum \Gamma \alpha_{(1)} \otimes y \alpha_{(2)}=\gamma \otimes y \alpha . \\
\Delta_{\mathcal{O}}(\beta)=\beta \otimes 1_{\mathcal{O}}+\gamma \otimes \beta
\end{array}\right.
\end{gathered}
$$

Since, by Proposition 1.13, $\tau(u h)=u \varepsilon_{H}(h)$ for every $u \in R, h \in H$, by applying the map $\tau: \mathcal{O} \rightarrow R: u \mapsto u_{(1)} S p\left(u_{(2)}\right)$ and a total integral $\lambda \in H^{*}$ (which exists as $H$ is cosemisimple), we get

$$
\left\{\begin{array} { l } 
{ \tau ( y \alpha _ { 1 } ) \otimes \alpha _ { 2 } = \tau ( y \alpha ) \otimes 1 _ { \mathcal { O } } } \\
{ \Gamma \alpha _ { 1 } \otimes y \alpha _ { 2 } = \gamma \otimes y \alpha } \\
{ 1 \lambda ( \beta ) = \beta + \gamma \lambda ( \beta ) , \lambda \in H ^ { * } }
\end{array} \quad \text { i.e. } \quad \left\{\begin{array}{l}
y \otimes \alpha=y \otimes \varepsilon_{H}(\alpha) 1_{\mathcal{O}} \\
\Gamma \alpha_{1} \otimes y \alpha_{2}=\gamma \otimes y \alpha \\
\beta=\lambda(\beta)(1-\gamma)
\end{array} \quad\right.\right. \text { i.e. }
$$




$$
\left\{\begin{array}{l}
\alpha=\varepsilon_{H}(\alpha) \in K \\
\Gamma \otimes y \alpha=\gamma \otimes y \alpha \\
\beta=\lambda(\beta)(1-\gamma)
\end{array} .\right.
$$

Now, since $z \notin H$ we have $\alpha \neq 0$ so that $\Gamma \otimes y \alpha=\gamma \otimes y \alpha$ is equivalent to $\Gamma \otimes y=\gamma \otimes y$ i.e. to $\gamma=\Gamma$. Then $z:=y \alpha+\lambda(\beta)(1-\Gamma), \alpha, \lambda(\beta) \in K$.

Proposition 4.8. Let $H$ be a Hopf algebra over a field $K$. Let $A$ be a bialgebra and let $\sigma: H \rightarrow A$ be an injective morphism of bialgebras having two retractions $\pi_{1}, \pi_{2}: A \rightarrow H$ (i.e. $\pi_{i} \sigma=\operatorname{Id}_{H}, i=1,2$ ) that are $H$-bilinear coalgebra maps. Denote by $R^{i}$ the pre-bialgebra in ${ }_{H}^{H} \mathcal{Y D}$ associated to $\left(A, \pi_{i}, \sigma\right)$ for $i=1,2$. For $i=1,2$ set $\tau_{i}: A \rightarrow R^{i}: a \mapsto a_{(1)} \sigma S \pi_{i}\left(a_{(2)}\right)$. Then

$$
\tau_{1 \mid}: R^{2} \rightarrow R^{1} \quad \text { and } \quad \tau_{2 \mid}: R^{1} \rightarrow R^{2}
$$

are mutual inverse coalgebra homomorphisms.

Proof. For every $u \in R^{2}$, since, by Proposition 1.13, $\tau_{i}[v \sigma(h)]=\tau_{i}(v) \varepsilon_{H}(h)$ for every $v \in A, h \in$ $H$, we get

$$
\tau_{2} \tau_{1}(u)=\tau_{2}\left[u_{(1)} \sigma S_{H} \pi_{1}\left(u_{(2)}\right)\right]=\tau_{2}\left(u_{(1)}\right) \varepsilon_{H} S_{H} \pi_{1}\left(u_{(2)}\right)=\tau_{2}(u)=u
$$

so that $\tau_{2 \mid} \circ \tau_{1 \mid}=\operatorname{Id}_{R^{2}}$. Similarly one proves that $\tau_{1 \mid} \circ \tau_{2 \mid}=\operatorname{Id}_{R^{1}}$. Let us check that $\tau_{1 \mid}$ is a coalgebra homomorphism. Let $u \in R^{2}$. We have

$$
\begin{aligned}
& \left(\tau_{1} \otimes \tau_{1}\right) \delta_{2}(u) \stackrel{(16)}{=} \sum \tau_{1}\left(\tau_{2}\left(u_{(1)}\right)\right) \otimes \tau_{1}\left(u_{(2)}\right) \\
= & \sum \tau_{1}\left(u_{(1)} \sigma S \pi_{2}\left(u_{(2)}\right)\right) \otimes \tau_{1}\left(u_{(3)}\right)=\sum \tau_{1}\left(u_{(1)}\right) \otimes \tau_{1}\left(u_{(2)}\right)=\delta_{1} \tau_{1}(u)
\end{aligned}
$$

where the last equality follows as $\tau_{i}: A \rightarrow R^{i}$ is a cpalgebra homomorphism and $\delta_{i}$ denotes the comultiplication of $R^{i}$. Moreover $\varepsilon_{1} \tau_{1}(u)=\varepsilon_{A}(u) \stackrel{16)}{=} \varepsilon_{1}(u)$.

TheOREM 4.9. Let $q$ be a primitive $N$-th root of unity. Let $(H, g, \chi, \lambda(N))$ be a compatible datum for $q$ where $H$ is a cosemisimple Hopf algebra. We use the notations of Theorem 4.2. Let

$$
\pi: \mathcal{O}(H, g, \chi, \lambda(N)) \rightarrow H
$$

an $H$-bilinear coalgebra homomorphism which is a retraction of the canonical injection $\sigma_{\mathcal{O}}$. Then $\pi=p$.

Proof. Set $\mathcal{O}:=\mathcal{O}(H, g, \chi, \lambda(N))$ and $\sigma:=\sigma_{\mathcal{O}}$. As observed in Definitions 1.11, there is a bialgebra isomorphism $\omega: R^{\pi} \#_{\zeta} H \rightarrow \mathcal{O}$ where $R^{\pi}$ is the pre-bialgebra in ${ }_{H}^{H} \mathcal{Y} \mathcal{D}$ associated to $(\mathcal{O}, \pi, \sigma)$ with corresponding cocycle $\zeta$.

Set $R^{p}:=R$. By Proposition 4.8

$$
\tau_{p \mid}: R^{\pi} \rightarrow R^{p}: u \mapsto u_{(1)} \sigma S p\left(u_{(2)}\right) \quad \text { and } \quad \tau_{\pi \mid}: R^{p} \rightarrow R^{\pi}: u \mapsto u_{(1)} \sigma S \pi\left(u_{(2)}\right)
$$

are mutual inverse coalgebra homomorphisms.

Note that $R^{p}$ is always thin unless $N=1$ and in this case $\sigma_{\mathcal{O}}$ is an isomorphism so that $\pi=\sigma_{\mathcal{O}}^{-1}=p$. Therefore we may assume that $R^{p}$ is thin. As a consequence $R^{\pi}$ is thin too. Set

$$
z:=\tau_{\pi}(y) \text {. }
$$

Clearly $P\left(R^{\pi}\right)=K z$. Let $\gamma \in H$ and $\theta \in H^{*}$ be such that $(H, \gamma, \theta)$ is the Yetter-Drinfeld datum associated to $z$ and let $q^{\pi}=\theta(\gamma)$. In view of Corollary 2.15, we have that:

a) There exists $\nu(N) \in K$ such that $(H, \gamma, \theta, \nu(N))$ is a compatible datum for $q$ and $z^{\cdot \mathcal{O}} N=$ $\nu(N)\left(1_{H}-\sigma(\gamma)^{N}\right)$.

b) The $n$-th iterated power of $z$ in $R^{\pi}$ and the $n$-th iterated power of $z$ in $\mathcal{O}$ coincides for every $0 \leq n \leq N-1$.

c) $R^{\pi}=R_{q}(H, \gamma, \theta)$ is a quantum line.

Moreover, $\mathcal{O} \simeq R^{\pi} \#_{\zeta} H$ as a bialgebra where, for any $a, b \in \mathbb{N}$, we get

$$
\zeta\left(z^{a} \otimes z^{b}\right)= \begin{cases}1 & \text { for } a+b=0 \\ \nu(N)\left(1_{H}-\gamma^{N}\right) & \text { for } a+b=N, a \neq 0, b \neq 0 \\ 0 & \text { otherwise. }\end{cases}
$$


We have $\omega\left(z \otimes 1_{H}\right)=z$ so that $\omega^{-1}(z)=z \otimes 1_{H}$ and hence

$$
\begin{aligned}
& \Delta_{\mathcal{O}}(z)=\Delta_{\mathcal{O}}\left(\omega \omega^{-1}(z)\right)=(\omega \otimes \omega) \Delta_{R^{\pi} \#_{\zeta} H}\left(\omega^{-1}(z)\right)=(\omega \otimes \omega) \Delta_{R^{\pi} \#_{\zeta} H}\left(z \otimes 1_{H}\right) \\
& =(\omega \otimes \omega)\left(\sum z^{(1)_{\pi}} \otimes z_{\langle-1\rangle}^{(2)_{\pi}} \otimes z_{\langle 0\rangle}^{(2)_{\pi}} \otimes 1_{H}\right) \\
& \stackrel{z \in P\left(R^{\pi}\right)}{=} \quad(\omega \otimes \omega)\left(z \otimes\left(1_{R}\right)_{\langle-1\rangle} \otimes\left(1_{R}\right)_{\langle 0\rangle} \otimes 1_{H}+1_{R} \otimes z_{\langle-1\rangle} \otimes z_{\langle 0\rangle} \otimes 1_{H}\right) \\
& \stackrel{(*)}{=} \quad(\omega \otimes \omega)\left(z \otimes 1_{H} \otimes 1_{R} \otimes 1_{H}+1_{R} \otimes \gamma \otimes z \otimes 1_{H}\right) \\
& =\quad z \otimes 1_{\mathcal{O}}+\sigma(\gamma) \otimes z,
\end{aligned}
$$

where in $(*)$ we used the colinearity of the unity map of $R^{\pi}$ and the formula $z_{\langle-1\rangle} \otimes z_{\langle 0\rangle}=\gamma \otimes z$ which holds as $(H, \gamma, \theta)$ is the Yetter-Drinfeld datum associated to $z$. By Lemma 4.7, we have that $\sigma(\gamma)=\Gamma=\sigma(g)$ (so that $\gamma=g$ ) and $z:=\alpha y+\beta(1-\Gamma)$ for some $\alpha, \beta \in K$. Hence

$$
z=\tau_{\pi}(z)=\tau_{\pi}[\alpha y+\beta(1-\Gamma)]=\alpha \tau_{\pi}(y)=\alpha z
$$

so that, since $z \neq 0$, we obtain $\alpha=1$.

Let us prove that $z=y$. Recall that $\Gamma y=q y \Gamma$. Set $a:=\beta(1-\Gamma) \in K\langle\Gamma\rangle$. For every $h \in K\langle\Gamma\rangle$ there exists $h^{\prime} \in K\langle\Gamma\rangle$ such that $h y=y h^{\prime}$ so that we can write

$$
\nu(N)\left(1_{H}-\Gamma^{N}\right)=z^{N}=(y+a)^{N}=y^{N}+a^{N}+y b=\lambda(N)\left(1_{\mathcal{O}}-\Gamma^{N}\right)+a^{N}+y b
$$

where $b=\sum_{i=0}^{N-2} y^{i} b_{i}$ and $b_{i} \in K\langle\Gamma\rangle$. Since $\left\{y^{\cdot{ }^{\circ}} \sigma(h) \mid 0 \leq i \leq N-1, h \in \mathcal{B}(H)\right\}$, is a basis for $\mathcal{O}$, we get $b=0$ and

$$
[\nu(N)-\lambda(N)]\left(1_{H}-\Gamma^{N}\right)=a^{N}=\beta^{N}\left(1_{\mathcal{O}}-\Gamma\right)^{N}=\sum_{i=0}^{N}\left(\begin{array}{c}
N \\
i
\end{array}\right) \beta^{N}(-\Gamma)^{i} .
$$

If $\Gamma$ has finite order $t$, then $\Gamma^{t}=1$ and hence $q^{t}=1$. From this we deduce $N=o(q) \mid t$. We have two cases.

$t=N)$ In this case $\Gamma^{N}=1$ so that

$$
0=[\nu(N)-\lambda(N)]\left(1_{H}-\Gamma^{N}\right)=\beta^{N}(-1)^{N}+\sum_{i=0}^{N-1}\left(\begin{array}{c}
N \\
i
\end{array}\right) \beta^{N}(-\Gamma)^{i} .
$$

The coefficient of $\Gamma^{N-1}$ is zero. Since $N \neq 1$ ( $R$ is thin) this coefficient is exactly $\left(\begin{array}{c}N \\ N-1\end{array}\right) \beta^{N}=N \beta^{N}$ and we obtain $\beta=0$.

$t \neq N)$ In this case $t>N$ and $1, \Gamma, \Gamma^{2}, \ldots, \Gamma^{N-1}, \Gamma^{N}$ are linearly independent in $K\langle\Gamma\rangle$ so that the coefficient of $\Gamma^{N-1}$ is zero. Since this coefficient is exactly $\left(\begin{array}{c}N \\ N-1\end{array}\right) \beta^{N}=N \beta^{N}$ we obtain $\beta=0$.

The same argument works when $\Gamma$ has infinite order.

In any case we have $z=\alpha y+\beta(1-\Gamma)=y$ so that $\nu(N)=\lambda(N)$.

Finally, for every $0 \leq i \leq N-1, h \in \mathcal{B}(H)$, by Proposition 1.13, we have

$$
\pi\left[y^{\cdot \mathcal{O}^{i}} \sigma(h)\right]=\pi\left(y^{\cdot \mathcal{O}^{i}}\right) h=\pi\left(z^{\cdot \mathcal{O}} i\right) h \stackrel{(a)}{=} \pi\left(z^{\mathcal{R}^{\pi} i}\right) h=\varepsilon_{\mathcal{R}^{\pi}}\left(z^{\mathcal{R}^{\pi} i}\right) h=\delta_{i, 0} h=p\left[y^{\cdot \mathcal{O}^{i}} \sigma(h)\right] .
$$

Thus $\pi=p$.

Corollary 4.10. Let $q$ be a primitive $N$-th root of unity. Let $(H, g, \chi, \lambda(N))$ be a compatible datum for $q$ where $H$ is a cosemisimple Hopf algebra. We use the notations of Theorem 4.9. Let $Q$ be a pre-bialgebra in ${ }_{H}^{H} \mathcal{Y D}$ with cocycle $\zeta$ such that there is a bialgebra isomorphism $\Phi: \mathcal{O}=$ $\mathcal{O}(H, g, \chi, \lambda(N)) \rightarrow Q \#_{\zeta} H$ where $\Phi \circ \sigma_{\mathcal{O}}$ is the canonical injection $H \hookrightarrow Q \#_{\zeta} H$. Then $\Phi$ induces an isomorphism

$$
\phi:\left(R_{q}(H, g, \chi), \xi\right) \rightarrow(Q, \zeta)
$$

of pre-bialgebras with a cocycle in ${ }_{H}^{H} \mathcal{Y D}$.

Proof. Let $\pi: Q \#_{\zeta} H \rightarrow H$ be the canonical projection. Then in view of Theorem 4.9, we have $\pi \circ \Phi=p$, where $p$ is the map defined in Theorem 4.2. By Proposition 1.15, $\Phi$ induces an isomorphism $\phi:\left(R_{q}(H, g, \chi), \xi\right) \rightarrow(Q, \zeta)$ of pre-bialgebras with a cocycle in ${ }_{H}^{H} \mathcal{Y D}$. 
Corollary 4.11. Let $H$ be a cosemisimple Hopf algebra over a field $K$. Let $A$ be a bialgebra and let $\sigma: H \rightarrow A$ be an injective morphism of bialgebras. Assume that there exists a retraction $\pi: A \rightarrow H$ (i.e. $\pi \sigma=H$ ) that is an $H$-bilinear coalgebra map and such that the coalgebra in the pre-bialgebra in ${ }_{H}^{H} \mathcal{Y D}$ associated to $(A, \pi, \sigma)$ is thin. Let $\pi^{\prime}: A \rightarrow H$ be an $H$-bilinear coalgebra homomorphism which is a retraction of the canonical injection $\sigma$. Then $\pi^{\prime}=\pi$.

Proof. Assume there exists a retraction $\pi: A \rightarrow H$ as in the statement.

By Theorem 4.4, there exist a primitive $N$-th root of unit $q, g \in H, \chi \in H^{*}$ and $\lambda(N) \in K$ such that $(H, g, \chi, \lambda(N))$ is a compatible datum for $q$ and there is a bialgebra isomorphism $\widehat{\sigma}$ : $\mathcal{O}(H, g, \chi, \lambda(N)) \rightarrow A$ such that $\widehat{\sigma} \circ \sigma_{\mathcal{O}}=\sigma$. Assume there is a retraction $\pi^{\prime}$ as in the statement. Then

$$
\pi \circ \widehat{\sigma} \circ \sigma_{\mathcal{O}}=\pi \circ \sigma=\operatorname{Id}_{H} \quad \text { and } \quad \pi^{\prime} \circ \widehat{\sigma} \circ \sigma_{\mathcal{O}}=\pi^{\prime} \circ \sigma=\operatorname{Id}_{H} .
$$

In view of Theorem 4.9 we have that $\pi \circ \widehat{\sigma}=p=\pi^{\prime} \circ \widehat{\sigma}$ so that $\pi=\pi^{\prime}$.

\section{Compatible Data}

In this section we include some results on compatible data that will be needed in the sequel.

LEMMA 5.1. Let $q$ be a primitive $N$-th root of unity and let $(H, g, \chi, \lambda(N))$ be a compatible datum for q. Let $E$ be a Hopf subalgebra of $H$ containing $K G(H)$. Then $\left(E, g, \chi_{\mid E}, \lambda(N)\right)$ is a compatible datum for $q$.

Proof. It is straightforward.

LEMMA 5.2. Let $q$ be a primitive $N$-th root of unity, where $N \neq 1$.

Let $(H, g, \chi, \lambda(N))$ be a compatible datum for $q$. We use the notations of Theorem 4.7. Let $\mathcal{O}=$ $\mathcal{O}(H, g, \chi, \lambda(N))$. Then, for every character $\eta \in \mathcal{O}^{*}$, one has $\eta(y)=0$. Moreover $\eta(\Gamma)^{N}=1_{K}$, whenever $\lambda(N) \neq 0$.

Proof. We will apply Theorem 4.2. Let us check that $\eta(y)=0$.

From $\Gamma y=y \varphi_{H}(\Gamma)=q y \Gamma$, by applying $\eta$ on both sides, we get $\eta(\Gamma y)=q \eta(y \Gamma)$ i.e. $\eta(\Gamma) \eta(y)=$ $q \eta(\Gamma) \eta(y)$ so that, since $q \neq 1(N \neq 1)$, one has $\eta(\Gamma) \eta(y)=0$. From $\eta(\Gamma) \neq 0$, we get $\eta(y)=0$. Let us prove that $\eta(\Gamma)^{N}=1_{K}$, whenever $\lambda(N) \neq 0$. We have

$$
0=\eta(y)^{N}=\eta\left(y^{N}\right)=\eta\left[\lambda(N)\left(1_{A}-\Gamma^{N}\right)\right]=\lambda(N)\left(1-\eta(\Gamma)^{N}\right)
$$

Since $\lambda(N) \neq 0$, we get $\eta(\Gamma)^{N}=1_{K}$.

LEMMA 5.3. Let $q$ be a primitive $N$-th root of unity and let $(H, g, \chi)$ be a Yetter-Drinfeld datum for $q$. Then $g \in Z(G(H))$ and $\chi \in Z\left(G\left(H^{*}\right)\right)$.

Proof. For any $a \in G(H)$, we have

$$
g \chi(a) a=g \sum \chi\left(a_{(1)}\right) a_{(2)} \stackrel{\$ 1}{\underline{8}} \sum a_{(1)} \chi\left(a_{(2)}\right) g=a \chi(a) g .
$$

Since $a$ is invertible, we have that $\chi(a) \neq 0$ and hence $g a=a g$.

Let $\gamma \in H^{*}$ be a character of $H$ and apply $\gamma$ to both sides of (8). We obtain

$$
\gamma(g) \sum \chi\left(h_{(1)}\right) \gamma\left(h_{(2)}\right)=\sum \gamma\left(h_{(1)}\right) \chi\left(h_{(2)}\right) \gamma(g)
$$

that is, since $\gamma(g) \neq 0$, that $\chi * \gamma=\gamma * \chi$.

Proposition 5.4. Let $q_{1}$ be a primitive $N_{1}$-th root of unity, $N_{1} \neq 0$

Let $\left(H^{1}, g_{1}, \chi_{1}, \lambda\left(N_{1}\right)\right)$ be a compatible datum for $q_{1}$. Using the notations of Theorem 4.2. let $H^{2}=\mathcal{O}\left(H^{1}, g_{1}, \chi_{1}, \lambda\left(N_{1}\right)\right)$ and set

$$
y_{1}=y, \quad \text { and } \quad \Gamma_{1}=\Gamma \text {. }
$$

Let $q_{2}$ be a primitive $N_{2}$-th root of unity, $N_{2} \neq 0$.

The following assertions are equivalent for a character $\chi_{2} \in\left(H^{2}\right)^{*}$, a group-like $\Gamma_{2} \in G\left(H^{2}\right)$ and an element $\lambda\left(N_{2}\right) \in K$ : 
(1) $\left(H^{2}, \Gamma_{2}, \chi_{2}, \lambda\left(N_{2}\right)\right)$ is a compatible datum for $q_{2}$

(2) $\left(H^{1}, \Gamma_{2}, \chi_{2 \mid H^{1}}, \lambda\left(N_{2}\right)\right)$ is a compatible datum for $q_{2}$,

$$
\chi_{2}\left(y_{1}\right)=0, \quad \chi_{2}\left(\Gamma_{1}\right) \chi_{1}\left(\Gamma_{2}\right)=1_{K},
$$

and

Moreover we have that

$$
y_{1} \Gamma_{2}^{N_{2}}=\Gamma_{2}^{N_{2}} y_{1} \text {, whenever } \lambda\left(N_{2}\right) \neq 0 .
$$

$$
y_{1} \Gamma_{2}^{N_{2}}=\Gamma_{2}^{N_{2}} y_{1} \quad \text { iff } \quad \chi_{1}\left(\Gamma_{2}\right)^{N_{2}}=1_{K} .
$$

Proof. First of all observe that, by Theorem 4.2, $H^{2}$ is a Hopf algebra and $\mathcal{O}\left(H^{1}, g_{1}, \chi_{1}, \lambda\left(N_{1}\right)\right) \simeq$ $R_{q}\left(H^{1}, g_{1}, \chi_{1}\right) \#_{\xi} H^{1}$ where $R=R_{q}\left(H^{1}, g_{1}, \chi_{1}\right)$ is a quantum line so that (see e.g. AMStu, Theorem 3.9]) $\operatorname{Corad}\left(R \#_{\xi} H^{1}\right)=K \otimes \operatorname{Corad}\left(H^{1}\right)$ which means that $\operatorname{Corad}\left(H^{2}\right)=\operatorname{Corad}\left(H^{1}\right)$ (here we identify $H^{1}$ with its image in $H^{2}$ ). Thus, since Corad $\left(H^{2}\right) \subseteq H^{1}$, we have $G\left(H^{2}\right) \subseteq H^{1}$.

Consider the algebra homomorphisms

$$
\begin{array}{ll}
\varphi_{H^{2}}: & H^{2} \rightarrow H^{2}, \varphi_{H^{2}}(h)=\sum \chi_{2}\left(h_{(1)}\right) h_{(2)}, \\
\psi_{H^{2}}: & H^{2} \rightarrow H^{2}, \psi_{H^{2}}(h)=\sum h_{(1)} \chi_{2}\left(h_{(2)}\right) .
\end{array}
$$

Let us prove that

$$
\Gamma_{2} \varphi_{H^{2}}\left(y_{1}\right)=\psi_{H^{2}}\left(y_{1}\right) \Gamma_{2} \Longleftrightarrow \chi_{2}\left(\Gamma_{1}\right) \chi_{1}\left(\Gamma_{2}\right)=1_{K} .
$$

In view of Lemma 5.2, $\chi_{2}\left(y_{1}\right)=0$ so that we have

$$
\begin{aligned}
& \varphi_{H^{2}}\left(y_{1}\right)=\sum \chi_{2}\left[\left(y_{1}\right)_{(1)}\right]\left(y_{1}\right)_{(2)}=\chi_{2}\left(y_{1}\right) 1_{A}+\chi_{2}\left(\Gamma_{1}\right) y_{1}=\chi_{2}\left(\Gamma_{1}\right) y_{1}, \\
& \psi_{H^{2}}\left(y_{1}\right)=\sum\left(y_{1}\right)_{(1)} \chi_{2}\left[\left(y_{1}\right)_{(2)}\right]=y_{1} \chi_{2}\left(1_{A}\right)+\Gamma_{1} \chi_{2}\left(y_{1}\right)=y_{1} .
\end{aligned}
$$

Thus, by the definition of the algebra structure of $H^{2}=\mathcal{O}$, we have

$$
\begin{aligned}
& \Gamma_{2} \varphi_{H^{2}}\left(y_{1}\right)=\chi_{2}\left(\Gamma_{1}\right) \Gamma_{2} y_{1}=\chi_{2}\left(\Gamma_{1}\right) y_{1} \varphi_{H^{1}}\left(\Gamma_{2}\right)=\chi_{1}\left(\Gamma_{2}\right) \chi_{2}\left(\Gamma_{1}\right) y_{1} \Gamma_{2}, \\
& \psi_{H^{2}}\left(y_{1}\right) \Gamma_{2}=y_{1} \Gamma_{2} .
\end{aligned}
$$

Thus

$$
\Gamma_{2} \varphi_{H^{2}}\left(y_{1}\right)=\psi_{H^{2}}\left(y_{1}\right) \Gamma_{2} \Longleftrightarrow \chi_{1}\left(\Gamma_{2}\right) \chi_{2}\left(\Gamma_{1}\right) y_{1} \Gamma_{2}=y_{1} \Gamma_{2} \Longleftrightarrow \chi_{2}\left(\Gamma_{1}\right) \chi_{1}\left(\Gamma_{2}\right)=1_{K} .
$$

Let us prove that

$$
\sum\left(y_{1}\right)_{(1)}\left(1_{H^{1}}-\Gamma_{2}^{N_{2}}\right) S\left[\left(y_{1}\right)_{(2)}\right]=0 \Longleftrightarrow y_{1} \Gamma_{2}^{N_{2}}=\Gamma_{2}^{N_{2}} y_{1} .
$$

We have

$$
\begin{aligned}
& \sum\left(y_{1}\right)_{(1)}\left(1_{H}-\Gamma_{2}^{N_{2}}\right) S\left[\left(y_{1}\right)_{(2)}\right] \\
= & y_{1}\left(1_{H}-\Gamma_{2}^{N_{2}}\right)+\Gamma_{1}\left(1_{H}-\Gamma_{2}^{N_{2}}\right) S\left(y_{1}\right)=y_{1}\left(1_{H}-\Gamma_{2}^{N_{2}}\right)-\Gamma_{1}\left(1_{H}-\Gamma_{2}^{N_{2}}\right) \Gamma_{1}^{-1} y_{1} .
\end{aligned}
$$

Thus

$$
\begin{aligned}
\sum\left(y_{1}\right)_{(1)}\left(1_{H^{1}}-\Gamma_{2}^{N_{2}}\right) S\left[\left(y_{1}\right)_{(2)}\right] & =0 \Longleftrightarrow y_{1}\left(1_{H}-\Gamma_{2}^{N_{2}}\right)=\Gamma_{1}\left(1_{H}-\Gamma_{2}^{N_{2}}\right) \Gamma_{1}^{-1} y_{1} \\
& \Longleftrightarrow y_{1} \Gamma_{2}^{N_{2}}=\Gamma_{1} \Gamma_{2}^{N_{2}} \Gamma_{1}^{-1} y_{1} \Longleftrightarrow y_{1} \Gamma_{2}^{N_{2}}=\Gamma_{2}^{N_{2}} y_{1} .
\end{aligned}
$$

The last equivalence follows from Lemma 5.3 which gives $\Gamma_{1} \in Z\left(G\left(H^{1}\right)\right)$ and hence $\Gamma_{1} \Gamma_{2}=\Gamma_{2} \Gamma_{1}$.

Let us prove (37). Since $\Gamma_{2} \in H^{1}$, we have

$$
\Gamma_{2}^{N_{2}} y_{1}=y_{1} \varphi_{H^{1}}\left(\Gamma_{2}^{N_{2}}\right)=\chi_{1}\left(\Gamma_{2}^{N_{2}}\right) y_{1} \Gamma_{2}^{N_{2}}=\chi_{1}\left(\Gamma_{2}\right)^{N_{2}} y_{1} \Gamma_{2}^{N_{2}}
$$

so that we get (37).

$(1) \Rightarrow(2)$. Since $\left(H^{2}, \Gamma_{2}, \chi_{2}, \lambda\left(N_{2}\right)\right)$ is a compatible datum for $q_{2}$, by Lemma 5.1, $\left(H^{1}, \Gamma_{2}, \chi_{2 \mid H^{1}}, \lambda\left(N_{2}\right)\right)$ is a compatible datum for $q_{2}$.

Since $\chi_{2}^{i}$ is a character, by Lemma 5.2, $\chi_{2}^{i}\left(y_{1}\right)=0$, for every $i \in \mathbb{N} \backslash\{0\}$. 
Since $\left(H^{2}, \Gamma_{2}, \chi_{2}\right)$ is a Yetter-Drinfeld datum for $q_{2}$, one has $\Gamma_{2} \varphi_{H^{2}}(h)=\psi_{H^{2}}(h) \Gamma_{2}$, for every $h \in H^{2}$. We apply this relation to the case $h=y_{1}: \Gamma_{2} \varphi_{H^{2}}\left(y_{1}\right)=\psi_{H^{2}}\left(y_{1}\right) \Gamma_{2}$. By (38), this condition is equivalent to $\chi_{2}\left(\Gamma_{1}\right) \chi_{1}\left(\Gamma_{2}\right)=1_{K}$.

Assume now $\lambda\left(N_{2}\right) \neq 0$. In this case we have

$$
\Gamma_{2}^{N_{2}} \neq 1_{H^{2}}, \quad \text { and } \quad \chi_{2}^{N_{2}}(h)\left(1_{H^{2}}-\Gamma_{2}^{N_{2}}\right)=\sum h_{(1)}\left(1_{H^{2}}-\Gamma_{2}^{N_{2}}\right) S h_{(2)}, \text { for every } h \in H^{2} .
$$

For $h=y_{1}$, we get

$$
0=\chi_{2}^{N_{2}}\left(y_{1}\right)\left(1_{H}-\Gamma_{2}^{N_{2}}\right)=\sum\left(y_{1}\right)_{(1)}\left(1_{H}-\Gamma_{2}^{N_{2}}\right) S\left[\left(y_{1}\right)_{(2)}\right]
$$

so that, by (39), $y_{1} \Gamma_{2}^{N_{2}}=\Gamma_{2}^{N_{2}} y_{1}$.

$(2) \Rightarrow(1)$. Let us prove that $\left(H^{2}, \Gamma_{2}, \chi_{2}\right)$ is a Yetter-Drinfeld datum for $q_{2}$ that is

$$
\Gamma_{2} \sum \chi_{2}\left(h_{(1)}\right) h_{(2)}=\sum h_{(1)} \chi_{2}\left(h_{(2)}\right) \Gamma_{2} \text {, for every } h \in H^{2} .
$$

Thus we have to prove that $\Gamma_{2} \varphi_{H^{2}}(h)=\psi_{H^{2}}(h) \Gamma_{2}$, for every $h \in H^{2}$.

Now, recall that

$$
W=\left\{y_{1}^{a} \sigma(h) \mid 0 \leq a \leq N_{1}-1, h \in \mathcal{B}\left(H^{1}\right)\right\}
$$

is a basis for $H^{2}$ so that it is enough to prove that

$$
\Gamma_{2} \varphi_{H^{2}}\left(y_{1}^{a} h\right)=\psi_{H^{2}}\left(y_{1}^{a} h\right) \Gamma_{2} \text {, for every } h \in \mathcal{B}\left(H^{1}\right) .
$$

Since $\varphi_{H^{2}}$ and $\psi_{H^{2}}$ are algebra homomorphisms, it is enough to check it for $a=1$ and $h=1_{H}$. In fact since $\left(H^{1}, \Gamma_{2}, \chi_{2 \mid H^{1}}\right)$ is a Yetter-Drinfeld datum for $q_{2}$, then we know that $\Gamma_{2} \varphi_{H^{2}}(h)=$ $\psi_{H^{2}}(h) \Gamma_{2}$, for every $h \in H^{1}$. Since $\chi_{2}\left(\Gamma_{1}\right) \chi_{1}\left(\Gamma_{2}\right)=1_{K}$, by (38), we have $\Gamma_{2} \varphi_{H^{2}}\left(y_{1}\right)=\psi_{H^{2}}\left(y_{1}\right) \Gamma_{2}$. Hence $\left(H^{2}, \Gamma_{2}, \chi_{2}\right)$ is a Yetter-Drinfeld datum for $q_{2}$.

Now, if $\lambda\left(N_{2}\right)=0$, then $\left(H^{1}, \Gamma_{2}, \chi_{2}, \lambda\left(N_{2}\right)\right)$ is a compatible datum.

Therefore we can assume $\lambda\left(N_{2}\right) \neq 0$ which implies

$$
\Gamma_{2}^{N_{2}} \neq 1_{H^{1}}, \quad \text { and } \quad \chi_{2 \mid H^{1}}^{N_{2}}(h)\left(1_{H^{1}}-\Gamma_{2}^{N_{2}}\right)=\sum h_{(1)}\left(1_{H^{1}}-\Gamma_{2}^{N_{2}}\right) S h_{(2)} \text {, for every } h \in H^{1} .
$$

Since $\lambda\left(N_{2}\right) \neq 0$ and $\left(H^{1}, \Gamma_{2}, \chi_{2 \mid H^{1}}, \lambda\left(N_{2}\right)\right)$ is a compatible datum for $q_{2}$, it remains to prove that

$$
\chi_{2}^{N_{2}}(h)\left(1_{H^{1}}-\Gamma_{2}^{N_{2}}\right)=\sum h_{(1)}\left(1_{H^{1}}-\Gamma_{2}^{N_{2}}\right) S h_{(2)} \text {, for every } h \in H^{2} \backslash H^{1},
$$

Assume there is $h \in H^{2} \backslash H^{1}$ such that

$$
\chi_{2}^{N_{2}}(h)\left(1_{H^{1}}-\Gamma_{2}^{N_{2}}\right) \neq \sum h_{(1)}\left(1_{H^{1}}-\Gamma_{2}^{N_{2}}\right) S h_{(2)} .
$$

Since $W$ is a basis for $H^{2}$, we can assume $h \in W$ and hence $h \in W \backslash H^{1}$ so that there exists a least $a \in \mathbb{N} \backslash\{0\}$ such that $h=y^{a} k$ for a suitable $k \in \mathcal{B}\left(H^{1}\right)$. We have

$$
\begin{aligned}
& \sum h_{(1)}\left(1_{H^{1}}-\Gamma_{2}^{N_{2}}\right) S h_{(2)} \\
& =\sum\left(y_{1}^{a} k\right)_{(1)}\left(1_{H^{1}}-\Gamma_{2}^{N_{2}}\right) S\left[\left(y_{1}^{a} k\right)_{(2)}\right] \\
& =\sum\left(y_{1}^{a}\right)_{(1)} k_{(1)}\left(1_{H^{1}}-\Gamma_{2}^{N_{2}}\right) S\left(k_{(2)}\right) S\left[\left(y_{1}^{a}\right)_{(2)}\right] \\
& \stackrel{k \in H^{1}}{=} \sum\left(y_{1}^{a}\right)_{(1)} \chi_{2}^{N_{2}}(k)\left(1_{H^{1}}-\Gamma_{2}^{N_{2}}\right) S\left[\left(y_{1}^{a}\right)_{(2)}\right] \\
& =\chi_{2}^{N_{2}}(k) \sum\left(y_{1}^{a}\right)_{(1)}\left(1_{H^{1}}-\Gamma_{2}^{N_{2}}\right) S\left[\left(y_{1}^{a}\right)_{(2)}\right] \\
& =\chi_{2}^{N_{2}}(k) \sum\left(y_{1}^{a-1} y_{1}\right)_{(1)}\left(1_{H^{1}}-\Gamma_{2}^{N_{2}}\right) S\left[\left(y_{1}^{a-1} y_{1}\right)_{(2)}\right] \\
& =\chi_{2}^{N_{2}}(k) \sum\left(y_{1}^{a-1}\right)_{(1)}\left(y_{1}\right)_{(1)}\left(1_{H^{1}}-\Gamma_{2}^{N_{2}}\right) S\left[\left(y_{1}\right)_{(2)}\right] S\left[\left(y_{1}^{a-1}\right)_{(2)}\right]=0 .
\end{aligned}
$$

In fact, since $\Gamma_{2}^{N_{2}} y_{1}=y_{1} \Gamma_{2}^{N_{2}}$, by (39), we have

$$
\sum\left(y_{1}\right)_{(1)}\left(1_{H^{1}}-\Gamma_{2}^{N_{2}}\right) S\left[\left(y_{1}\right)_{(2)}\right]=0 .
$$


Let us prove that $\chi_{2}^{n}(y)=0$ for every $n \in \mathbb{N}$.

If $n=0$ then $\chi_{2}^{n}\left(y_{1}\right)=\varepsilon\left(y_{1}\right)=0$.

If $n=1$ then $\chi_{2}^{n}\left(y_{1}\right)=\chi_{2}\left(y_{1}\right)=0$.

Let $n \geq 1$ and assume $\chi_{2}^{i}\left(y_{1}\right)=0$ for every $0 \leq i \leq n-1$. We have

$$
\chi_{2}^{n}\left(y_{1}\right)=\left(\chi_{2}^{n-1} \otimes \chi_{2}\right) \Delta_{H^{2}}\left(y_{1}\right)=\chi_{2}^{n-1}\left(y_{1}\right) \chi_{2}\left(1_{A}\right)+\chi_{2}^{n-1}\left(\Gamma_{1}\right) \chi_{2}\left(y_{1}\right)=0 .
$$

Moreover, we have

$$
\chi_{2}^{N_{2}}(h)\left(1_{H^{1}}-\Gamma_{2}^{N_{2}}\right)=\chi_{2}^{N_{2}}\left(y^{a} k\right)\left(1_{H^{1}}-\Gamma_{2}^{N_{2}}\right)=\left[\chi_{2}^{N_{2}}(y)\right]^{a} \chi_{2}^{N_{2}}(k)\left(1_{H^{1}}-\Gamma_{2}^{N_{2}}\right)=0 .
$$

Hence

a contradiction.

$$
\chi_{2}^{N_{2}}(h)\left(1_{H^{1}}-\Gamma_{2}^{N_{2}}\right)=\sum\left(h_{1}\right)_{(1)}\left(1_{H^{1}}-\Gamma_{2}^{N_{2}}\right) S\left[\left(h_{1}\right)_{(2)}\right]
$$

\section{EXAMPLES}

First of all we want to exhibit an example of a bosonization as in Definitions 1.10 which is not a Radford-Majid bosonization.

EXAmple 6.1. Let $N>1$. Assume that the field $K$ is algebraically closed. Let $H=K\langle g\rangle$, where $\langle g\rangle$ is the multiplicative group associated to $\mathbb{Z}$. Let $q \in K$ be a primitive $N$-th root of unity. Let $\chi \in H^{*}$ be the character of $H$ defined by setting $\chi(g)=q$.

Then $\chi^{N}=\varepsilon_{H}$. Let now $\lambda(N)$ be an arbitrary non-zero element of $K$.

Then, by Remark 3.2 we can apply Proposition 3.5 and deduce that, $(H, g, \chi, \lambda(N))$ is a compatible datum for $q$. By Theorem 4.2 there exists a bialgebra $\mathcal{O}=\mathcal{O}(H, g, \chi, \lambda(N))$, an injective bialgebra map $\sigma: H \rightarrow \mathcal{O}$ and an element $y \in \mathcal{O}$ such that

$$
\left\{y^{a} \Gamma^{n} \mid 0 \leq a \leq N-1, n \in \mathbb{Z}\right\}
$$

is a basis for $\mathcal{O}$. Moreover the algebra structure of $\mathcal{O}$ is given by $y^{N}=\lambda(N)\left(1_{\mathcal{O}}-\Gamma^{N}\right), \Gamma^{n} y^{a}=$ $q^{a n} y^{a} \Gamma^{n}$ for any $a \in \mathbb{N}$, and $n \in \mathbb{Z}$, and the coalgebra structure is given by $\Delta_{\mathcal{O}}(y)=y \otimes 1_{\mathcal{O}}+\Gamma \otimes y$. Here $\Gamma=\sigma(g)$. Furthermore

$$
\omega: R_{q}(H, g, \chi) \#_{\xi} H \rightarrow \mathcal{O}(H, g, \chi, \lambda(N)), \omega(r \otimes h)=r \sigma_{\mathcal{O}}(h)
$$

is a Hopf algebra isomorphism where

$$
\xi\left(y^{a} \otimes y^{b}\right)= \begin{cases}1 & \text { for } a+b=0 \\ \lambda(N)\left(1_{H}-g^{N}\right) & \text { for } a+b=N, a \neq 0, b \neq 0 \\ 0 & \text { otherwise. }\end{cases}
$$

and $R_{q}(H, g, \chi)$ is an $N$-dimensional quantum line spanned by the powers of $y$ and the $N$-th power of $y$ in $R$ is zero. Since the compatible datum is non-trivial, by Theorem 2.14, $\mathcal{O}$ is not a Radford-Majid bosonization.

REMARK 6.2. Note that, by Corollary 4.10, there exists essentially a unique pre-bialgebra $Q$ in ${ }_{H}^{H} \mathcal{Y D}$ with cocycle $\zeta$ such that there is a bialgebra isomorphism $\Phi: \mathcal{O}=\mathcal{O}(H, g, \chi, \lambda(N)) \rightarrow$ $Q \#{ }_{\zeta} H$ where $\Phi \circ \sigma_{\mathcal{O}}$ is the canonical injection $H \hookrightarrow Q \#{ }_{\zeta} H$. Thus $\mathcal{O}$ can not be regarded as a Radford-Majid bosonization of its Hopf subalgebra $H$.

Remark 6.3. Let $H$ be a Hopf algebra over a field $K$. Let $A$ be a bialgebra and let $\sigma: H \rightarrow A$ be an injective morphism of bialgebras having a retraction $\pi: A \rightarrow H$ (i.e. $\pi \sigma=H$ ) that is an $H$-bilinear coalgebra map. Assume that $H$ is f.d. and that the coalgebra in the pre-bialgebra in ${ }_{H}^{H} \mathcal{Y D}$ associated to $(A, \pi, \sigma)$ is $N$-dimensional and thin.

Then, by Theorem 4.4, there exists a primitive $N$-th root of unit $q, g \in H, \chi \in H^{*}, \lambda(N) \in K$ such that $(H, g, \chi, \lambda(N))$ is a compatible datum for $q$ and there is a bialgebra isomorphism $\widehat{\sigma}$ : $\mathcal{O}(H, g, \chi, \lambda(N)) \rightarrow A$ such that $\widehat{\sigma} \circ \sigma_{\mathcal{O}}=\sigma$. We are looking for a minimal example of this situation such that the coalgebra underlying the pre-bialgebra $R$ in ${ }_{H}^{H} \mathcal{Y D}$ associated to $(A, \pi, \sigma)$ is not a quantum line. In view of Theorem 2.14, we have that $N$ is even and $\xi\left(y \otimes y^{\cdot R}{ }^{N / 2-1}\right) \neq 0$. Note that the assumptions of 2.2 are fulfilled so that we can keep also the notations therein. By (29) in 
Proposition 2.8, we have $\xi\left(y \otimes y^{N / 2-1}\right)=(N / 2-1)_{q} ! x$ so that $\xi\left(y \otimes y^{N / 2-1}\right)=0$ is equivalent to $x=0$. Thus we need $N$ even and $x \neq 0$. In view of Lemma 2.6, from $x \neq 0$, we obtain $N / 2 \neq 1$ odd and $H$ not cosemisimple.

Then the minimal candidate for $N / 2$ is 3 and hence $N=6$.

Note that $N \mid \operatorname{dim} H$. In fact $N \mid o(g)$ and $o(g) \mid \operatorname{dim} K G(H)$. If $N=\operatorname{dim} H$ then $H=K\langle g\rangle$ and hence $H$ is cosemisimple. Since this should not be the case, we need $N \neq \operatorname{dim} H$ and hence the minimal candidate is $\operatorname{dim} H=2 N=12$. Thus

$$
\operatorname{dim} A=\operatorname{dim} R \cdot \operatorname{dim} H=6 \cdot 12=72 .
$$

We now give an example of a 72-dimensional Hopf algebra $A$ with a 12-dimensional Hopf subalgebra $H$ and an $H$-bilinear coalgebra projection $\pi$ onto $H$ which is a retraction of the canonical injection $\sigma$. The coalgebra underlying the pre-bialgebra $R$ in ${ }_{H}^{H} \mathcal{Y D}$ associated to $(A, \pi, \sigma)$ is a thin coalgebra but not a quantum line. In fact the multiplication is not left $H$-colinear (see also Theorem 2.14). We have that $R$ is generated as an algebra by the powers of $y$, where $P(R)=K y$, and that the powers of $y$ in $A$ and the powers of $y$ in $R$ are actually different. This example shows that Corollary 4.11 can fail whenever we drop the assumption of $H$ being a cosemisimple Hopf algebra. In fact, in view of AMStu, Theorem 4.2] $A$ admits an $H$-bilinear projection $p: A \rightarrow H$ such $p \sigma=\operatorname{Id}_{H}$ but $p \neq \pi$ (the pre-bialgebra associated to $p$ is a quantum line).

EXAMPLE 6.4. Let $K$ be a field containing primitive 6 -th root of unity $q$. Let $H^{1}=K G$ where $G=\langle\gamma\rangle$ is the cyclic group of order 6 . Let $\chi_{1}: G \rightarrow K$ be the character of $G$ defined by setting $\chi_{1}(\gamma)=-1$ and extend, by linearity, $\chi_{1}$ to a character of $H^{1}$. Let

$$
N_{1}=2, \quad q_{1}=-1, \quad H^{1}=k G, \quad g_{1}=\gamma^{3}, \quad \lambda\left(N_{1}\right)=0 \in K
$$

Then $\left(H^{1}, g_{1}, \chi_{1}, \lambda\left(N_{1}\right)\right)$ is a compatible datum for $q_{1}$. By applying Theorem 4.2 we obtain a Hopf algebra $A^{1}=\mathcal{O}\left(H^{1}, g_{1}, \chi_{1}, \lambda\left(N_{1}\right)\right)$, generated as an algebra by a group like element $\gamma$ of order 6 and a skew primitive element $x$ with the following relations (where we identify $H^{1}$ with its image in $\left.A^{1}\right)$ :

$$
\begin{gathered}
\gamma^{6}=1_{A^{1}}, \quad x^{2}=0, \quad \gamma x+x \gamma=0, \\
\Delta_{A^{1}}(\gamma)=\gamma \otimes \gamma, \varepsilon_{A^{1}}(\gamma)=1_{A^{1}}, \quad \Delta_{A^{1}}(x)=\gamma^{3} \otimes x+x \otimes 1_{A^{1}}, \quad \varepsilon_{A^{1}}(x)=0, \\
S_{A^{1}}(\gamma)=\gamma^{-1}, \quad S_{A^{1}}(x)=-\gamma^{-3} x .
\end{gathered}
$$

This 12-dimensional Hopf algebra is called $\mathcal{B}_{0}$ (see $[\mathrm{Na}]$ )

Let $H^{2}=A^{1}=\mathcal{O}\left(H^{1}, g_{1}, \chi_{1}, \lambda\left(N_{1}\right)\right)$ and set

$$
\Gamma_{1}=\gamma^{3}
$$

Let

$$
\begin{array}{ll}
N_{2}=6, & q_{2}=q=\text { primitive } 6 \text {-th root of unity } \\
\Gamma_{2}=\gamma, & \lambda\left(N_{2}\right)=0 \in K
\end{array}
$$

Let $\chi_{2}: H^{2} \rightarrow K$ be the character defined by $\chi_{2}(\gamma)=q_{2}$, and $\chi_{2}(x)=0$. By Proposition 5.4, in order to prove that $\left(H^{2}, \Gamma_{2}, \chi_{2}, \lambda\left(N_{2}\right)\right)$ is a compatible datum for $q_{2}$, it is enough to check that $\left(H^{1}, \Gamma_{2}, \chi_{2 \mid H^{1}}, \lambda\left(N_{2}\right)\right)$ is a compatible datum for $q_{2}$ and that

$$
\chi_{2}(x)=0, \quad \chi_{2}\left(\Gamma_{1}\right) \chi_{1}\left(\Gamma_{2}\right)=1_{K} .
$$

Since $\lambda\left(N_{2}\right)=0$ we have only to prove that $\left(H^{1}, \Gamma_{2}, \chi_{2 \mid H^{1}}\right)$ is a Yetter-Drinfeld datum for $q_{2}$ and that $\chi_{2}\left(\Gamma_{1}\right) \chi_{1}\left(\Gamma_{2}\right)=1_{K}$. We have

$$
\chi_{2}\left(\Gamma_{1}\right) \chi_{1}\left(\Gamma_{2}\right)=\chi_{2}\left(\gamma^{3}\right) \chi_{1}(\gamma)=q^{3}(-1)=-q^{3}=1 .
$$

Moreover $\chi_{2 \mid H^{1}}\left(\Gamma_{2}\right)=\chi_{2 \mid H^{1}}(\gamma)=\chi_{2}(\gamma)=q_{2}$. We have to prove that

$$
\gamma \sum \chi_{2 \mid H^{1}}\left(h_{(1)}\right) h_{(2)}=\sum h_{(1)} \chi_{2 \mid H^{1}}\left(h_{(2)}\right) \gamma, \text { for every } h \in H^{1}
$$


Consider the morphisms

$$
\varphi_{H^{1}}: H^{1} \rightarrow H^{1}: h \longmapsto \sum \chi_{2 \mid H^{1}}\left(h_{(1)}\right) h_{(2)}, \quad \psi_{H^{1}}: H^{1} \rightarrow H^{1}: h \longmapsto \sum h_{(1)} \chi_{2 \mid H^{1}}\left(h_{(2)}\right) .
$$

Note that both $\varphi_{H^{1}}$ and $\psi_{H^{1}}$ are algebra homomorphism so that, in order to prove that $\gamma \varphi_{H^{1}}(h)=$ $\psi_{H^{1}}(h) \gamma$, for every $h \in H^{1}$ it is enough to check it for $h=\gamma$.

Since $\varphi_{H^{1}}(\gamma)=q \gamma=\psi_{H^{1}}(\gamma)$, we get that $\left(H^{2}, \Gamma_{2}, \chi_{2}, \lambda\left(N_{2}\right)\right)$ is a compatible datum for $q_{2}$.

We apply Theorem 4.2. Therefore there exists a Hopf algebra $A^{2}=\mathcal{O}\left(H^{2}, \Gamma_{2}, \chi_{2}, \lambda\left(N_{2}\right)\right)$, an injective Hopf algebra map $\sigma: H^{2} \rightarrow A^{2}$ and an element $y \in A^{2}$ such that

$$
\left\{y^{i} \sigma(h) \mid 0 \leq i \leq N-1, h \in \mathcal{B}\left(H^{2}\right)\right\}
$$

is a basis for $A^{2}$ where $\mathcal{B}\left(H^{2}\right)$ is a basis of $H^{2}$. Let

$$
\Gamma=\sigma\left(\Gamma_{2}\right), \quad X=\sigma(x) \quad \text { and } \quad Y=y .
$$

Then the algebra structure of $A^{2}$ is given by

$$
\begin{aligned}
Y^{6} & =0, \quad \Gamma^{6}=1_{A^{2}}, \quad X^{2}=0, \\
\Gamma Y & =q Y \Gamma, \quad X Y=-Y X, \quad \Gamma X=-X \Gamma,
\end{aligned}
$$

and coalgebra structure given by

$$
\begin{aligned}
\Delta_{A^{2}}(Y) & =Y \otimes 1_{A^{2}}+\Gamma \otimes Y, \\
\Delta_{A^{2}}(X) & =\Gamma^{3} \otimes X+X \otimes 1_{A^{2}}, \quad \Delta_{A^{2}}(\Gamma)=\Gamma \otimes \Gamma .
\end{aligned}
$$

Now we simply write $H=H^{2}$ and $A=A^{2}$. Identify $H$ with $\sigma(H)$. Define

$$
\pi: A \rightarrow H, \pi\left(Y^{i} h\right)=\delta_{i, 0} h+\delta_{i, 3} X h,
$$

for any $0 \leq i \leq 5, h \in H$. It is straightforward to prove that $\pi$ is an $H$-bilinear retraction of $\sigma$.

Let us prove that $\pi$ is a morphism of coalgebras. Since $\pi$ is $H$-bilinear, it is enough to check it on the powers of $Y$. We have

$$
(\Gamma \otimes Y)\left(Y \otimes 1_{A}\right)=\Gamma Y \otimes Y=q Y \Gamma \otimes Y=q\left(Y \otimes 1_{A}\right)(\Gamma \otimes Y) .
$$

By the quantum binomial formula, for any $0 \leq n \leq 5$, we deduce

$$
\Delta_{A}\left(Y^{n}\right)=\sum_{i=0}^{n}\left(\begin{array}{c}
n \\
i
\end{array}\right)_{q} Y^{n-i} \Gamma^{i} \otimes Y^{i}
$$

so that

$$
\begin{aligned}
(\pi \otimes \pi) \Delta_{A}\left(Y^{n}\right) & =\delta_{n, 0} 1_{H} \otimes 1_{H}+\delta_{n, 3} X \otimes 1_{H}+\delta_{n, 3} \Gamma^{3} \otimes X=\Delta_{H} \pi\left(Y^{n}\right) \\
\varepsilon_{H} \pi\left(Y^{i} h\right) & =\varepsilon_{H}\left(\delta_{i, 0} h+\delta_{i, 3} X h\right)=\delta_{i, 0} \varepsilon_{H}(h)=\varepsilon_{A}\left(Y^{i} h\right) .
\end{aligned}
$$

We want to compute

$$
R=A^{C o(H)}=\left\{a \in A \mid \sum a_{(1)} \otimes \pi\left(a_{(2)}\right)=a \otimes 1_{H}\right\} .
$$

Let $0 \leq n \leq 5$. Using the quantum binomial formula we obtain

$$
\sum\left(Y^{n}\right)_{(1)} \otimes \pi\left[\left(Y^{n}\right)_{(2)}\right]=Y^{n} \otimes 1_{H}+\left(\begin{array}{l}
n \\
3
\end{array}\right)_{q} Y^{n-3} \Gamma^{3} \otimes X
$$

Since $\left(\begin{array}{l}n \\ 3\end{array}\right)_{q}=0$ for $0 \leq n \leq 2$, we get $1_{A}, Y, Y^{2} \in R$. Recall from Proposition 1.13, that the map $\tau: A \rightarrow R, \tau(a)=\sum a_{(1)} \sigma S_{H} \pi\left[a_{(2)}\right]$ defines a coalgebra homomorphism such that $\tau(a \sigma(h))=$ $\tau(a) \varepsilon_{H}(h)$. We have

$$
\tau\left(Y^{n}\right)=Y^{n}+\left(\begin{array}{l}
n \\
3
\end{array}\right)_{q} Y^{n-3} \Gamma^{3} S_{H}(X)=Y^{n}-\left(\begin{array}{l}
n \\
3
\end{array}\right)_{q} Y^{n-3} X .
$$

In particular we get

$$
\begin{aligned}
\tau(1) & =1, \quad \tau(Y)=Y, \quad \tau\left(Y^{2}\right)=Y^{2}, \quad \tau\left(Y^{3}\right)=Y^{3}-X, \\
\tau\left(Y^{4}\right) & =Y^{4}-(2 q-1) Y X, \quad \tau\left(Y^{5}\right)=Y^{5}+Y^{2} X .
\end{aligned}
$$


Since $\tau\left(Y^{n}\right)=Y^{n}-\left(\begin{array}{l}n \\ 3\end{array}\right)_{q} Y^{n-3} X$ and

$$
\left\{Y^{i} \sigma(h) \mid 0 \leq i \leq N-1, h \in \mathcal{B}\left(H^{2}\right)\right\}
$$

is a basis for $A$, we get that $\left\{\tau\left(Y^{n}\right) \mid 0 \leq n \leq 5\right\}$ is linearly independent over $K$. Since $\tau$ is surjective and $\tau(a \sigma(h))=\tau(a) \varepsilon_{H}(h)$, we deduce that $\left\{\tau\left(Y^{n}\right) \mid 0 \leq n \leq 5\right\}$ generates $R$ over $K$ and hence it is a basis. Let $y=\tau(Y)=Y$ and denote by $y^{n}$ the $n$-th iterated power of $y$ in $R$. Since (see Proposition 1.13 $\tau(a) \cdot R \tau(b)=\tau[\tau(a) \cdot A b]$, for every $a, b \in A$, it is easy to prove that

$$
y^{n}=\tau\left(Y^{n}\right)=Y^{n}-\left(\begin{array}{l}
n \\
3
\end{array}\right)_{q} Y^{n-3} X, \text { for every } 0 \leq n \leq 5 .
$$

From this, since $y=Y$, we get that the powers of $Y$ in $A$ and the powers of $Y$ in $R$ are actually different. Moreover $R$ is generated over $K$ by $\left\{y^{n} \mid 0 \leq n \leq 5\right\}$.

Note that, in view of Theorem $4.2 A=\mathcal{O}\left(H, \Gamma_{2}, \chi_{2}, \lambda\left(N_{2}\right)\right)$ has an $H$-bilinear projection $p: A \rightarrow H$ such that $p \sigma=\operatorname{Id}_{H}$. Moreover the underlying coalgebra structure $Q$ of the prebialgebra in ${ }_{H}^{H} \mathcal{Y D}$ associated to $(A, p, \sigma)$ is a thin coalgebra. In view of Proposition 4.8, $R$ is isomorphic as a coalgebra to $Q$ so that $R$ is thin too. In particular $P(R)=K y$.

By Theorem 2.14, we get that the multiplication of $R$ is not left $H$-colinear. Let us check this directly. Since, for every $r \in R, \rho(r)=\sum \pi\left(r_{(1)}\right) \otimes r_{(2)}$, we obtain

$$
\rho_{A}\left(Y^{n}\right)=\Gamma^{n} \otimes Y^{n}+\left(\begin{array}{l}
n \\
3
\end{array}\right)_{q} X \Gamma^{n-3} \otimes Y^{n-3} \quad \text { and } \quad \rho_{A}(Y X)=\Gamma^{4} \otimes Y X+\Gamma X \otimes Y
$$

so that

$$
\rho\left(y^{4}\right)=\rho_{A}\left(Y^{4}-\left(\begin{array}{l}
4 \\
3
\end{array}\right)_{q} Y X\right)=\Gamma^{4} \otimes y^{4}+2\left(\begin{array}{l}
4 \\
3
\end{array}\right)_{q} X \Gamma \otimes y
$$

Since $\rho\left(y^{2}\right)=\Gamma^{2} \otimes y^{2}$ we infer that the multiplication of $R$ is not left $H$-colinear. Finally, since, for any $r, s \in R$, we have $\xi(r \otimes s)=\pi\left(r \cdot{ }_{A} s\right)$, we obtain $\xi\left(y \otimes y^{2}\right)=\pi\left(Y \cdot{ }_{A} Y^{2}\right)=\pi\left(Y^{3}\right)=X$ so that $\xi$ is not trivial.

Remark 6.5. Let $H$ be a Hopf algebra over a field $K$. Let $A$ be a bialgebra and let $\sigma: H \rightarrow A$ be an injective morphism of bialgebras having a retraction $\pi: A \rightarrow H$ (i.e. $\pi \sigma=H$ ) that is an $H$-bilinear coalgebra map. Assume that either $H$ is f.d. and that the coalgebra underlying the pre-bialgebra in ${ }_{H}^{H} \mathcal{Y D}$ associated to $(A, \pi, \sigma)$ is $N$-dimensional and thin.

Then, by Theorem 4.4, there exists a primitive $N$-th root of unit $q, g \in H, \chi \in H^{*}, \lambda(N) \in$ $K$ such that $(H, g, \chi, \lambda(N))$ is a compatible datum for $q$ and there is a bialgebra isomorphism $\widehat{\sigma}: \mathcal{O}(H, g, \chi, \lambda(N)) \rightarrow A$ such that $\widehat{\sigma} \circ \sigma_{\mathcal{O}}=\sigma$. We are looking for a minimal example of this situation such that $R$ is a quantum line but the bosonization is not a Radford-Majid bosonization. In view of Theorem 2.14, this means $\lambda(N) \neq 0$.

Note that $N \mid \operatorname{dim} H$. If $\operatorname{dim} H=p$ a prime number, then $N$ is either 1 or $p$. In the first case $\xi=\varepsilon \otimes \varepsilon$ so that the bosonization is trivial. In the second case $g^{N}=1_{H}$. By definition of compatible datum we get $\lambda(N)=0$.

Hence the minimal candidate is $\operatorname{dim} H=4$. Let $H=K C_{4}$ where $C_{4}=\langle g\rangle$ is the multiplicative cyclic group of order 4 . Set $N=2$. Let $\chi: H \rightarrow K$ be the character defined by setting $\chi(g)=-1$. Then, $(H, g, \chi)$ is a Yetter-Drinfeld datum for the primitive $N$-th root of the unity $q=-1$. By Proposition 3.5, for every $\lambda(N) \in K,(H, g, \chi, \lambda(N))$ is a compatible datum for $q=-1$. Thus, for every $\lambda(N) \in K \backslash\{0\}, \mathcal{O}(H, g, \chi, \lambda(N))$ is the example we were looking for.

\section{REFERENCES}

[AS] N. Andruskiewitsch, H.-J. Schneider, Lifting of quantum linear spaces and pointed Hopf algebras of order $p^{3}$, J. Algebra 209 (1998), 658-691.

[AMSte] A. Ardizzoni, C. Menini and D. Stefan, A Monoidal Approach to Splitting Morphisms of Bialgebras, Trans. Amer. Math. Soc., 359 (2007), 991-1044.

[AMStu] A. Ardizzoni, C. Menini and F. Stumbo, Small Bialgebras with Projection, J. Algebra (2007), doi:10.1016/j.jalgebra.2007.04.008. 
[CDMM $]$ C. Călinescu, S. Dăscălescu, A. Masuoka, C. Menini, Quantum lines over non-cocommutative cosemisimple Hopf algebras, J. Algebra 273 (2004), 753-779.

[Maj] S. Majid, Crossed products by braided groups and bosonization, J. Algebra 163 (1994), 165-190.

[Na] S. Natale, Hopf algebras of dimension 12, Algebr. Represent. Theory 5 (2002), 445-455.

[Rad] D. E. Radford, The Structure of Hopf Algebras with a Projection, J. Algebra 92 (1985), 322-347.

[Scha] P. Schauenburg, The structure of Hopf algebras with a weak projection, Algebr. Represent. Theory 3 (2000), 187-211.

University of Ferrara, Department of Mathematics, Via Machiavelli 35, Ferrara, I-44100, Italy

E-mail address: alessandro.ardizzoni@unife.it

$U R L:$ http://www.unife.it/utenti/alessandro.ardizzoni

University of Ferrara, Department of Mathematics, Via Machiavelli 35, Ferrara, I-44100, Italy

E-mail address: men@dns.unife.it

URL: http://www.unife.it/utenti/claudia.menini 